\title{
Méthodologie interdisciplinaire de prévention des TMS : association de la Biomécanique et de la Clinique de l'activité
}

Interdisciplinary methodological framework of biomechanics and occupational psychology for MSD prevention

Adriana Savescu, Pascal Simonet, Clarisse Gaudez et Gabriel Fernandez

\section{OpenEdition} Journals

Édition électronique

URL : https://journals.openedition.org/activites/5802

DOI : $10.4000 /$ activites.5802

ISSN : 1765-2723

Éditeur

ARPACT - Association Recherches et Pratiques sur les ACTivités

\section{Référence électronique}

Adriana Savescu, Pascal Simonet, Clarisse Gaudez et Gabriel Fernandez, « Méthodologie

interdisciplinaire de prévention des TMS : association de la Biomécanique et de la Clinique de

l'activité ", Activités [En ligne], 17-2 | 2020, mis en ligne le 15 octobre 2020, consulté le 25 août 2021.

URL : http://journals.openedition.org/activites/5802 ; DOI : https://doi.org/10.4000/activites.5802

Ce document a été généré automatiquement le 25 août 2021.

\section{c) (†)}

Activités est mis à disposition selon les termes de la licence Creative Commons Attribution - Pas d'Utilisation Commerciale - Pas de Modification 4.0 International. 


\section{Méthodologie interdisciplinaire de prévention des TMS : association de la Biomécanique et de la Clinique de l'activité}

Interdisciplinary methodological framework of biomechanics and occupational psychology for MSD prevention

Adriana Savescu, Pascal Simonet, Clarisse Gaudez et Gabriel Fernandez

\section{NOTE DE L'ÉDITEUR}

Article soumis le 8 janvier 2020, accepté le 19 juin 2020

Les auteurs remercient Olivier Morel et Gilles Reno pour leur implication et leur expertise technique et statistique.

\section{Introduction}

1 Les troubles musculosquelettiques (TMS) demeurent les maladies professionnelles les plus rencontrées dans de nombreux secteurs d'activités malgré les nombreuses actions réalisées pour les prévenir. Plusieurs revues de la littérature (Denis, St. Vincent, Jetté, Nastasia, \& Imbeau, 2005 ; Rivilis, Van Eerd, Cullen, Cole, Irvin, Ryson et al., 2008 ; Silverstein \& Clark, 2004) rendent compte de différents types d'intervention en les différenciant en fonction du type de transformations qu'elles génèrent: techniques, organisationnelles ou comportementales. Dans leur analyse bibliographique, Denis et al. (Denis et al., 2005) s'intéressent non seulement aux types de transformations réalisées, mais aussi aux modèles d'intervention mis en œuvre pour la prévention des TMS. Ils détaillent notamment un modèle d'intervention, largement diffusé, faisant l'objet d'un 
certain consensus (Aptel, Cail, \& Aublet-Cuvelier, 2014; St-Vincent, Chicoine, \& Beaugrand, 1998) qui comporte trois étapes principales: (1) identifier le problème de TMS dans l'entreprise, (2) réaliser un diagnostic des situations de travail sur lesquelles il est nécessaire d'intervenir en priorité (description du travail, évaluation du risque et identification de leurs déterminants (ou facteurs), (3) maîtriser le risque par la recherche de solutions et leurs implantations. Ce modèle d'intervention se base sur un modèle étiologique de TMS prenant en compte l'exposition des travailleurs aux facteurs de risque en lien avec l'organisation du travail et préconise, notamment, la réduction de l'exposition des opérateurs aux facteurs de risque identifiés. D'autres modèles étiologiques de survenue des TMS (Roquelaure, 2016), sous l'influence d'approches fondées sur les théories de l'activité de travail, prennent en compte le rôle actif de l'opérateur qui n'est plus considéré comme subissant l'exposition aux facteurs de risque, mais en capacité d'opérer des régulations dans son activité (Aptel \& Vézina, 2008 ; Vézina, 2001). Ces approches soutiennent un mécanisme de promotion de la santé qui vise à concilier une logique de réduction des expositions avec le développement des possibilités d'influence des opérateurs sur leurs situations de travail. Ainsi, le mécanisme d'exposition se complète d'hypothèses sur d'autres mécanismes psycho-physiopathologiques fondés sur la distinction entre «mouvement» et "geste» (Bourgeois \& Hubault, 2005; Clot \& Fernandez, 2005; Gaudez \& Aptel, 2008; Tomàs, 2013) en dépassant la composante physique des mouvements pour y intégrer des facteurs psychologiques, éducatifs, culturels et sociaux (Simonet, 2011). Dans les milieux professionnels, le geste est toujours contextualisé par le métier qui le façonne et qu'il façonne. Le geste est l'un des instruments de l'activité des professionnels. Il se fabrique dans les dilemmes et les tensions à l'œuvre entre les différents registres du métier : personnel, interpersonnel, transpersonnel et impersonnel ${ }^{1}$ (Simonet \& Clot, 2014). Le geste est aussi, par essence, social et psychologique. Il permet au sujet des actes de réalisation de la posture psychologique et sociale adressés à autrui. Il s'inscrit donc dans une histoire individuelle, collective et organisationnelle, et pour le réaliser, le sujet peut, plus ou moins selon les situations de travail, déployer des trésors d'imagination et d'ingéniosité (Pezé, 1998). On ne peut donc pas limiter l'étude du geste de métier et ses perspectives de développement aux seuls enchainements segmentaires efficaces et opératoires. Ainsi, une des préoccupations de prévention des TMS prenant en compte ces considérations est celle de la compréhension des gestes de métier tels qu'ils émergent et tels qu'ils se réinventent dans leur environnement de travail pour réaliser l'activité. L'approche par le geste interroge les pratiques des acteurs en santé au travail, dans leur capacité à construire des débats encadrés et organisés sur les questions de métier au sein de leurs dispositifs d'intervention Caroly, Coutarel, Escriva, Roquelaure, Schweitzer, \& Daniellou, 2008; Fernandez, 2009). Dans cette perspective développementale, suivant laquelle «le corps humain (est) prédisposé à une multiplicité indéfinie d'états, du plus défavorable au plus favorable à sa puissance d'agir, en passant par les plus neutres et les plus indifférents » (Jaquet, 2004), les travaux en clinique de l'activité permettent d'avancer que les TMS prennent largement leur source dans des gestes empêchés (Clot \& Fernandez, 2005). Ils sont définis comme des gestes qui se trouvent enfermés dans une répétition de nature compulsive quelle que soit la variabilité des obstacles rencontrés dans l'action quotidienne de travail. La voie choisie par la méthodologie clinique de l'activité est celle du dépassement du geste de métier empêché, prisonnier d'automatismes délétères, avec comme enjeu 
d'examiner les ressorts susceptibles - du côté des professionnels - de le transformer en geste de métier enrichi d'autres possibilités de réalisations (Fernandez, 2004 ; Simonet \& Caroly, 2008). Ainsi, l'idée suivie est que les organes au fonctionnement perturbé, empêché, recèlent des potentialités de développement dans leur confrontation aux obstacles du milieu (physique et social) (Vygotski, 1934/1994).

En ce qui concerne le mouvement (Gaudez \& Aptel, 2008), il a souvent été étudié seul dans de nombreux travaux sur les troubles musculo-squelettiques notamment pour évaluer les sollicitations biomécaniques des opérateurs. Le mouvement peut être caractérisé à l'aide de différentes techniques telles que l'observation (Brunet \& Riff, 2009) et la mesure de l'activité musculaire (par l'électromyographie de surface, technique non invasive) (Gaudez \& Cail, 2016 ; Gaudez, Wild, \& Aublet-Cuvelier, 2015). Ces deux techniques peuvent être utilisées en situation réelle de travail (Gaudez, Bonnet, \& Aublet-Cuvelier, 2013; Savescu, Gaudez, Aublet-Cuvelier, Simonet, Fernandez, Van-Trier et al., 2010) et apportent des informations présentant des niveaux de granularité différents. Les informations issues de l'électromyographie de surface apportent des informations concernant l'activité musculaire. Ainsi, pour un même mouvement observé, l'activité musculaire peut être différente sans pouvoir identifier les raisons. En complémentarité, l'analyse clinique de l'activité place les professionnels en situation d'observer et d'analyser les gestes et les dilemmes de l'activité et du métier auxquels ils doivent faire face au quotidien.

3 Prenant en compte l'approche par l'analyse du geste de métier et le caractère multifactoriel des TMS, la littérature scientifique encourage une analyse pluridisciplinaire de l'activité et des gestes afin de proposer des solutions de prévention plus adaptées aux besoins des entreprises (Vézina, 2001). En réalité, malgré des efforts réalisés (Plamondon \& Denis, 2008 ; Savescu \& Cuny-Guerrier, 2015 ; Thibault, Merlin, \& Garrigou, 2013), des avancées sont encore attendues sur la mise en pratique des interventions interdisciplinaires. En effet, le développement du geste de métier repose, en partie, sur des débats entre professionnels au sein de leur métier, mais aussi sur des débats avec les concepteurs des règles prescrites d'exercice du métier (Caroly et al., 2008 ; Massot \& Simonet, 2017). L'objectif de cet article est de montrer comment l'analyse du mouvement a pu devenir un outil pour enrichir la discussion entre professionnels sur le geste de métier dans une perspective de prévention des TMS. Cette action interdisciplinaire (Vinck, 2002) a été menée par deux équipes ayant la volonté de combiner leurs compétences: la clinique de l'activité en psychologie du travail et la biomécanique (Savescu \& Simonet, 2020). L'enjeu est de montrer comment l'analyse biomécanique participe à la création d'outils d'intervention mis en œuvre dans un cadre méthodologique en clinique de l'activité afin de permettre aux professionnels tout d'abord de mieux s'observer puis de s'en saisir pour mettre leur travail en discussion; de dépasser la barrière de la fatalité; de sortir de l'intersubjectivité pour, in fine, s'essayer à d'autres manières d'exécuter leurs gestes de métier et ainsi les développer en en faisant des « gestes dialogués » (Simonet \& Caroly, 2020). 


\section{Contexte global et cadre méthodologique de la demande initiale}

4 Cette intervention répond à la demande formulée par un service de médecine préventive préoccupé par la multiplication, dans le métier de fossoyeur municipal, des cas de TMS et de plaintes associées à ces pathologies (Van Trier, Simonet, Fernandez, Savescu, \& Gaudez, 2010) ${ }^{2}$. La dégradation constatée de l'état de santé des fossoyeurs et l'augmentation des taux d'absentéisme pour arrêt maladie compliquent l'organisation du service funéraire. Un constat est partagé entre la direction de ce service et la médecine du travail : « les aménagements de postes sont difficiles car toutes les tâches sollicitent les membres supérieurs et le rachis lombaire. Ils perturbent de plus l'organisation du travail et augmentent la charge de travail des fossoyeurs dont les postes ne sont pas aménagés. Ces derniers ayant un risque accru de TMS». Le reclassement dans un autre métier est souvent une impasse soit parce que le fossoyeur refuse de quitter son métier soit parce qu'il n'existe pas de poste disponible dans un autre service. La question qui finit par s'imposer est la suivante : comment un fossoyeur peut-il durer dans ce métier? Par ailleurs, le bilan des actions déjà menées en matière de prévention des TMS fait notamment état d'un décalage entre la formation " gestes et postures » dispensée aux fossoyeurs par un organisme spécialisé, extérieur à la municipalité, et les réalités complexes du métier.

5 La demande adressée à l'équipe de Psychologie du travail clinique de l'activité est motivée par le souhait des préventeurs, des personnels de direction et des membres du CHSCT (actuel Comité Social et Économique (CSE)) d'améliorer l'efficacité de la politique de prévention des TMS sur la base d'un examen approfondi de la complexité du métier de fossoyeur.

6 L'intervention est conduite sur plusieurs mois. Elle est structurée principalement autour de :

- trois collectifs de fossoyeurs de deux cimetières. Ils sont associés à l'intervention et représentent environ un tiers de l'effectif global des deux cimetières,

- un comité de pilotage réunissant les personnels de l'encadrement, de la médecine du travail et de la prévention des risques professionnels et incluant les intervenants-chercheurs,

- le CHSCT qui est informé à intervalles réguliers de l'avancée de l'intervention et qui a l'occasion d'en débattre.

Cette organisation de l'action permet de distinguer différents groupes homogènes de pairs (Oddone \& Briante, 1981) et temps d'échanges :

- entre fossoyeurs, sans participation directe de la hiérarchie, sur les critères qui signent, à leurs yeux, la qualité du service funéraire,

- entre les membres du comité de pilotage ${ }^{3}$ en charge de définir ensemble, à partir de logiques différentes, les actions de prévention des TMS et les questions liées à l'organisation du travail des fossoyeurs (Massot \& Simonet, 2017),

- entre les membres du CHSCT alimentés par les rapports d'activités des intervenants chercheurs et du médecin du travail

8 C'est après plusieurs mois d'une intervention conduite avec les méthodes d'analyse de l'activité mobilisées en ergonomie et en clinique de l'activité (Simonet, Caroly, \& Clot, 2011), qu'est apparue la nécessité d'ouvrir une coopération interdisciplinaire avec l'analyse biomécanique. Cette nouvelle coopération tente de répondre à la demande des 
fossoyeurs de mieux comprendre les liens entre les douleurs ressenties et l'exécution d'un geste précis : le jeté arrière.

Ce geste est réputé pour être particulièrement délicat : il est sollicitant pour le corps et certains fossoyeurs ne veulent pas le faire. Cependant, il est indispensable lors de la réalisation d'une fosse de 1,50 à $2 \mathrm{~m}$ de profondeur. Il consiste à sortir, de la fosse creusée, la terre en arrière de soi (sans pouvoir la mettre dans une brouette à proximité) en la projetant loin derrière par-dessus sa tête, son épaule droite ou gauche selon les habitudes de chacun. Ce geste est souvent associé pour les fossoyeurs et leur médecin du travail aux douleurs ressenties au niveau du bas du dos et des épaules. Ils expriment le souhait d'orienter davantage l'intervention sur l'examen de la complexité de ce geste.

Du point de vue de la démarche clinique de l'activité, et à cette étape de l'histoire de l'intervention, les enjeux de l'action interdisciplinaire avec la biomécanique sont principalement :

- du côté des fossoyeurs : les outiller avec des éléments d'observation et d'analyse en vue d'un examen plus approfondi, plus argumenté et plus controversé de la complexité de ce geste en rapport avec la mobilisation de leur corps dans l'activité et leur santé,

- du côté des membres du comité de pilotage et du $\mathrm{CHSCT}^{4}$ : nourrir leurs débats sur les questions d'organisation du travail, de choix du matériel, de politique de prévention des TMS sur la base d'une analyse plus approfondie des liens entre activité, corps au travail et santé.

11 La suite de cet article se concentre sur les modalités concrètes de réalisation de cette coopération interdisciplinaire qui complète les analyses précédentes et qui s'inscrit dans une perspective de développement du geste pour la prévention des TMS.

\section{Cadre méthodologique de l'action interdisciplinaire}

12 Afin de répondre à la nouvelle demande des fossoyeurs et de leur médecin du travail de poursuivre l'analyse du geste de jeté arrière, une méthodologie d'intervention interdisciplinaire a été élaborée. Elle associe la biomécanique et la clinique de l'activité et comporte 6 étapes :

1. discussions portant sur la faisabilité de la coopération puis de la construction du protocole de l'intervention entre les deux équipes: l'équipe de psychologie du travail clinique de l'activité du Conservatoire National d'Arts et Métiers (CNAM) et l'équipe de biomécanique du Département Homme au Travail de l'Institut National de Recherche et de Sécurité (INRS),

2. présentation d'un protocole d'enregistrement des sollicitations musculaires aux fossoyeurs et appel au volontariat pour le recueil de données,

3. recueil et analyse des données,

4. présentation des résultats de l'analyse aux fossoyeurs volontaires et émergence des premières discussions,

5. organisation d'auto-confrontations simples et croisées entre fossoyeurs volontaires sur la base des résultats de l'analyse biomécanique et des enregistrements vidéo,

6. analyse multimodale des auto-confrontations croisées (analyse croisée des énoncés langagiers et des gestes réalisés en auto-confrontations).

13 À chaque étape de la méthodologie les chercheurs des deux équipes étaient acteurs de la construction. Les efforts d'appropriation des méthodes de recueil ou d'analyse de 
données des uns et des autres ont été présents tout le long de la méthodologie interdisciplinaire.

\section{1. Éléments de discussions du protocole interdisciplinaire}

14 Le protocole d'étude de ce geste est destiné à produire des connaissances sur les dilemmes du métier souvent ignorés et dont les détails révélés permettent de penser autrement les actions et dispositifs de prévention des TMS. L'analyse biomécanique a pour fonction de renseigner un point aveugle de l'analyse clinique de l'activité : les sollicitations musculaires mobilisées dans la réalisation des différentes manières d'exécuter ce geste. Cette analyse biomécanique pourra alors permettre la conduite de nouvelles analyses par auto-confrontations simples et croisées enrichies par l'examen des rapports entre dilemmes de l'activité musculaire et dilemmes de l'activité concrète de travail constitutifs des dilemmes du métier de fossoyeur.

Pour atteindre ces objectifs, sans antériorité d'expérience sur ce type d'association interdisciplinaire, les points suivants sont discutés :

- décider d'agir ensemble, sans se mettre d'accord préalablement sur l'usage d'un vocabulaire scientifique commun, par exemple sur la définition des notions de geste, automatisme et mouvement, chacun partant de définitions appartenant à des champs disciplinaires différents,

- trancher le dilemme «mesures biomécaniques en laboratoire » ou « mesures biomécaniques sur le terrain ", avec des conséquences sur la conduite des opérations suivant l'option retenue,

- confirmer le choix du geste de jeté arrière parmi les gestes qui se discutent entre fossoyeurs, celui pour lequel l'analyse biomécanique peut être la plus efficace du point de vue du débat professionnel des fossoyeurs,

- se prêter, pour l'équipe de biomécanique, à un objectif qui va au-delà de la caractérisation de l'exécution du mouvement pour transmettre aux fossoyeurs des connaissances sur la variabilité intra- et inter-individuelle afin de lutter contre cette fatalité du métier «quand on est fossoyeur on est condamné à se faire mal »,

- opérer pour la clinique de l'activité un renouveau du cadre méthodologique installé chez les fossoyeurs en s'appuyant, notamment, sur des résultats tirés de l'analyse biomécanique pour conduire les auto-confrontations simples et croisées ( $\mathrm{cf} \S 2.5$ ).

\subsection{Présentation du protocole d'enregistrement des sollicitations biomécaniques}

Le choix de mesure en situation réelle de travail a été fait suivant une volonté d'être au plus proche des conditions de réalisation de l'activité.

Suite aux discussions entre les deux équipes de recherche et le choix de travailler sur le geste de jeté arrière ( $c f \S 2.1$.), des précautions sont prises afin d'introduire ce nouveau protocole d'analyse aux fossoyeurs. Cette étape a comme objectifs :

- d'engager une réflexion par le débat entre les fossoyeurs sur les liens entre ce geste identifié comme complexe et les questions de métier qui se posent pour réaliser le creusement d'une fosse,

- de leur présenter un possible protocole en vue de répondre à leur demande d'approfondissement de compréhension de ce geste, 
- de les mobiliser pour la phase d'enregistrement. muscles au niveau des épaules et du dos. Elle a été réalisée dans l'objectif de mettre en évidence la variabilité intra et inter-individuelle autour du geste de jeté arrière et de permettre aux fossoyeurs de "visualiser", de se rendre compte de cette variabilité, afin de s'en saisir dans leurs discussions à une étape ultérieure du protocole méthodologique interdisciplinaire. Le challenge est donc:

- de réaliser une intervention et un enregistrement comportant de l'électromyographie de surface dans des conditions réelles de travail (en plein air, dans une fosse) impliquant plusieurs fossoyeurs

- de pouvoir présenter les résultats biomécaniques sous une forme accessible aux fossoyeurs

- de créer un nouvel outil d'analyse de l'activité qui permette d'orienter les dialogues entre fossoyeurs sur cette dimension cachée de leur activité quotidienne de travail qui implique l'engagement de leur organisme comme instrument corporel de l'activité de travail

Cette opération de décontextualisation permet de créer les conditions d'une analyse du geste afin de conduire le débat sur des éléments qui restent habituellement dans l'ombre de l'analyse des conditions de travail.

\subsubsection{Matériels et méthode}

\subsubsection{Participants}

Huit fossoyeurs volontaires participent à cette phase de l'intervention, leur âge, taille, poids ainsi que leur expérience professionnelle et plus précisément comme fossoyeur sont présentés dans le tableau 1. Prenant en compte l'expérience en tant que fossoyeur, parmi les huit fossoyeurs participants, quatre sont débutants (avec une expérience comprise entre quelques mois et un an) et quatre sont expérimentés (avec une expérience comprise entre sept et trente-huit ans). Les participants à l'étude sont préalablement informés du déroulement de l'expérimentation, de la confidentialité des résultats et ont donné leur consentement pour y participer. 
Tableau 1 : Moyenne (écart type) et étendue de l'âge, de la taille, du poids, de l'expérience professionnelle et de l'expérience comme fossoyeur des 8 participants.

Table 1: Mean (standard deviation) age, height, weight, general professional experience and experience as gravedigger of the 8 participants of the study

\begin{tabular}{|c|c|c|}
\hline & $\begin{array}{l}\text { Moyenne } \\
\text { (écart type) }\end{array}$ & Etendue \\
\hline Age (ans) & $33,6(11,6)$ & $23-59$ \\
\hline Taille $(\mathrm{cm})$ & $176,4(9)$ & $163-188$ \\
\hline Poids (kg) & $79,2(19,2)$ & $60-124$ \\
\hline $\begin{array}{l}\text { Expérience } \\
\text { professionnelle (ans) }\end{array}$ & $16,4(12,8)$ & $8-45$ \\
\hline $\begin{array}{l}\text { Expérience comme } \\
\text { fossoyeur (ans) }\end{array}$ & $10,2(15,2)$ & $0,33-38$ \\
\hline
\end{tabular}

\subsubsection{Conditions expérimentales}

L'enregistrement des données électromyographiques a lieu en situation réelle de travail pour le creusement d'une fosse. Afin de standardiser la situation de travail lors de l'enregistrement des jetés arrières, les paramètres suivants sont imposés: emplacement du tas de terre derrière l'opérateur, creusement réalisé par un fossoyeur seul (sans l'aide d'un collègue), présence d'un coffrage pour retenir la terre expulsée, présence d'un chemin entre le coffrage et la fosse, réalisation de l'opération de creusement dans un emplacement du cimetière avec une terre plutôt grasse, dans une fosse d'une profondeur entre $1,5 \mathrm{~m}$ et $2 \mathrm{~m}$ sans contrainte temporelle (Figure 1 ). Tous ces paramètres sont identifiés préalablement avec les fossoyeurs lors des analyses antérieures de l'activité et lors des discussions préalables (cf $\$ 2.2$.). L'ensemble de ces paramètres correspond à une situation considérée comme la plus difficile pour l'exécution du geste de jeté arrière.

Figure 1 : Exemples d'images de jeté arrière. Figure 1: Examples of backward throwing

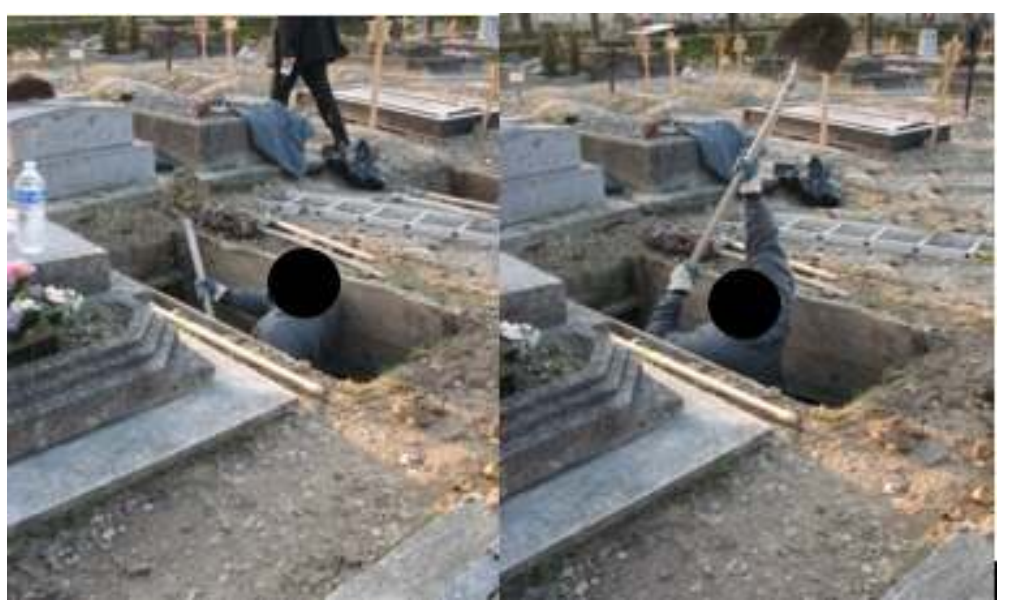

La consigne donnée aux fossoyeurs est de «travailler comme d'habitude en sortant la terre en jetés arrière » : sortir la terre de la fosse creusée en arrière de soi (sans pouvoir la mettre dans une brouette à proximité) en la projetant loin derrière par-dessus sa tête, son épaule droite ou gauche selon les habitudes de chacun. Les 8 fossoyeurs 
réalisent, lors de leur activité de creusement, des gestes de jeté arrière avec chacun de leurs outils de travail habituels (fourche, louchet ou pelle).

\subsubsection{Procédure de recueil de l'activité musculaire et enregistrement vidéo}

Les participants à l'étude sont instrumentés avec des moyens de mesure de l'activité musculaire de surface (électromyographie de surface (EMG)) utilisant un système d'enregistrement portable (ME3000 P8-1, Mega Electronics Ltd, Finlande) comportant des électrodes bipolaires avec une surface active de $6 \mathrm{~mm}$ (Ambu Blue Sensors ${ }^{\circ} \mathrm{N}-00-\mathrm{S} /$ 25, Denmark). Les électrodes sont collées sur la peau à une distance de $20 \mathrm{~mm}$ entre elles suivant les recommandations des auteurs du projet SENIAM (SENIAM5, 1997) et Zipp (Zipp, 1982). La peau est préalablement préparée: nettoyée avec un savon antiseptique et rasée au lieu de pose des électrodes avec un rasoir jetable pour éliminer les poils et la partie superficielle de la couche cornée de l'épiderme. L'activité électrique des muscles suivants est enregistrée: deltoïde antérieur droit et gauche, deltoïde moyen droit et gauche, deltoïde postérieur droit et gauche, lombaire (L3) droit et gauche. Le choix de ces muscles est en lien avec le geste de jeté arrière qui sollicite principalement les épaules et le dos. Une fois les données enregistrées, chaque signal EMG est amplifié (412 fois) et filtré $(5-800 \mathrm{~Hz}$ à une fréquence de $1000 \mathrm{~Hz})$. La valeur moyenne quadratique (root mean square - RMS) sur des intervalles consécutifs de $40 \mathrm{~ms}$ est calculée pour chaque enregistrement.

Pour chaque fossoyeur, une période de familiarisation avec l'instrumentation en situation de travail est effectuée avant l'enregistrement proprement dit. ${ }^{5}$

Pour la normalisation des données EMG, plusieurs méthodes sont préconisées dans la littérature (Burden, 2010 ; Fischer, Belbeck, \& Dickerson, 2010 ; Yang \& Winter, 1983) ainsi que différents tests (Boettcher, Ginn, \& Cathers, 2008 ; Kelly, Kadrmas, Kirkendall, \& Speer, 1996; Matkowski, Martin, \& Lepers, 2011; McCully, Kumar, Lazarus, \& Karduna, 2005 ; SENIAM5, 1997). Prenant en considération les douleurs manifestées par les fossoyeurs et les objectifs de l'étude, la normalisation des signaux EMG est faite en bilatéral à des efforts sous-maximaux. Les postures sont choisies selon la fonction principale du muscle et des recommandations de la littérature (McCully et al., 2005 ; SENIAM5, 1997) (Tableau 2). Elles sont maintenues et enregistrées pendant une période de 20 s. Pour l'enregistrement de l'EMG au repos, le sujet est assis les mains sur les genoux. La normalisation est réalisée suivant l'équation suivante :

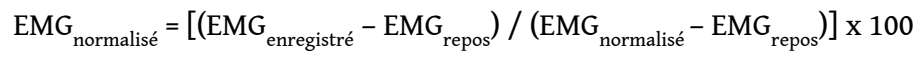


Tableau 2 : Procédure de normalisation des EMG des muscles enregistrés. Table 2: EMG normalization procedure for the recorded muscles

\begin{tabular}{|c|c|c|c|}
\hline Muscle & Poids & Test & Figure \\
\hline $\begin{array}{l}\text { Deltoïde } \\
\text { antérieur (droit } \\
\text { et gauche) }\end{array}$ & $\begin{array}{l}2 \mathrm{~kg} \text { en } \\
\text { chaque } \\
\text { main }\end{array}$ & $\begin{array}{l}\text { debout, les coudes fléchis } \\
\text { à } 90^{\circ} \text { et placés en avant à } \\
45^{\circ} \text { par rapport au tronc } \\
\text { dans le plan sagittal } \\
\text { (épaule à } 45^{\circ} \text { en flexion) }\end{array}$ & \\
\hline $\begin{array}{l}\text { Deltoïde moyen } \\
\text { (droit et gauche) }\end{array}$ & $\begin{array}{l}2 \mathrm{~kg} \text { en } \\
\text { chaque } \\
\text { main }\end{array}$ & $\begin{array}{l}\text { debout, les bras tendus et } \\
\text { maintenus en élévation à } \\
\text { hauteur des épaules de } \\
\text { chaque côté de leur corps } \\
\text { (plan frontal) }\end{array}$ & $0^{2 / 6}$ \\
\hline $\begin{array}{l}\text { Deltoïde } \\
\text { postérieur (droit } \\
\text { et gauche) }\end{array}$ & $\begin{array}{l}2 \mathrm{~kg} \text { en } \\
\text { chaque } \\
\text { main }\end{array}$ & $\begin{array}{l}\text { debout, les coudes fléchis } \\
\text { à } 90^{\circ} \text { et placés en arrière } \\
\text { à } 45^{\circ} \text { par rapport au tronc } \\
\text { dans le plan sagittal } \\
\text { (épaule à } 45^{\circ} \text { en } \\
\text { extension) }\end{array}$ & $8^{15^{\circ}}$ \\
\hline $\begin{array}{l}\text { Lombaire (droit } \\
\text { et gauche) }\end{array}$ & $\begin{array}{l}2 \mathrm{~kg} \text { en } \\
\text { chaque } \\
\text { main }\end{array}$ & $\begin{array}{l}\text { debout, les bras tendus et } \\
\text { maintenus en élévation à } \\
\text { l'horizontale devant (plan } \\
\text { sagittal) à hauteur des } \\
\text { épaules }\end{array}$ & $8_{2 k \mathbb{E}}^{2 x_{k}}$ \\
\hline
\end{tabular}

Les informations individuelles (taille, poids...) sont recueillies pour caractériser le groupe des volontaires. Par la normalisation de l'activité musculaire, les enregistrements sont rapportés aux caractéristiques de chaque individu. Cette opération rend possible l'analyse de la variabilité intra et inter-individuelle.

Un enregistrement vidéo synchrone à celui des EMG est réalisé. Il permet de visualiser à postériori l'activité des fossoyeurs en lien avec les signaux EMG et d'élaborer un film en vue des auto-confrontations.

\subsubsection{Analyse des données EMG}

Le mouvement de jeté arrière est défini de façon suivante (Figure 2) : il commence au moment où l'outil chargé de terre quitte le sol de la fosse, il finit quand la terre est expulsée de l'outil, elle n'est plus en contact avec l'outil. Ainsi, sont écartées du champ de l'analyse toutes les autres opérations du creusement. Un cycle correspond à un mouvement de jeté arrière ainsi défini. L'analyse est réalisée cycle par cycle et elle prend en compte l'expérience des fossoyeurs volontaires dans ce métier. 
Figure 2 : Définition d'un mouvement de jeté arrière suivant l'enregistrement synchronisé de l'EMG des muscles et de l'enregistrement vidéo.

Figure 2: Definition of the backward throwing movement based on EMG and video recordings

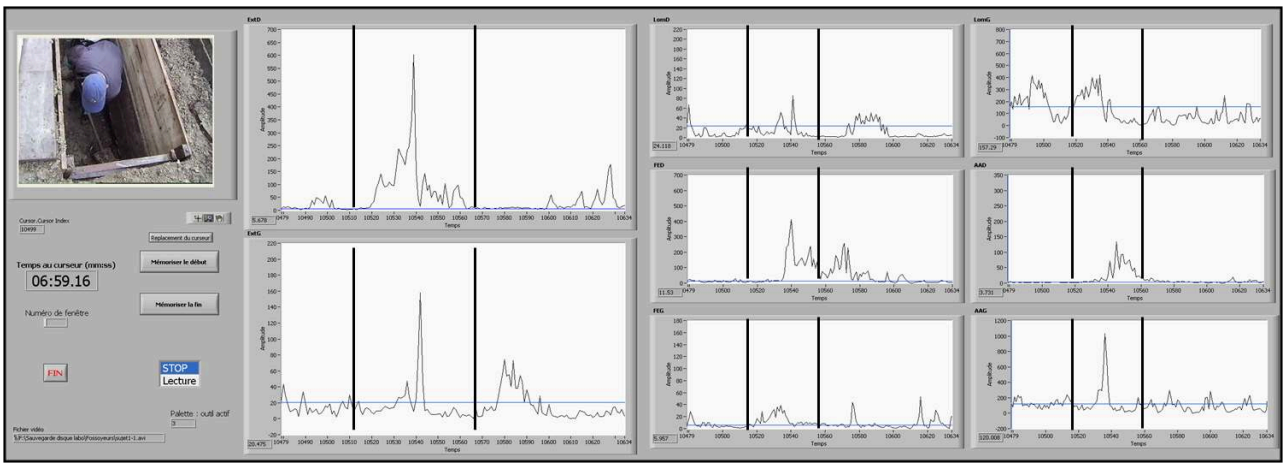

Les variables retenues pour l'analyse d'un cycle sont: le pic maximum (EMG max) de chacun des 8 muscles et la durée du cycle. Une comparaison entre les différents cycles est réalisée.

31 L'influence de l'expérience (novices/expérimentés) est analysée à l'aide d'une analyse one-way ANOVA utilisant comme variables dépendantes : le pic maximum d'EMG pour chaque muscle enregistré et la durée du cycle. Pour l'analyse de la variabilité intrasujet une analyse de régression est utilisée pour le pic maximum de l'EMG pour chaque muscle enregistré. Pour toutes les analyses, le seuil de significativité choisi est de $\mathrm{p}<0.05$.

\subsubsection{Résultats de l'analyse biomécanique}

Les analyses montrent une moyenne de l'EMG max significativement plus élevée pour les novices par rapport aux expérimentés pour 7 muscles sur les 8 enregistrés (Figure 3) : deltoïde antérieur droit, deltoïde latéral droit, deltoïde postérieur gauche, lombaire droit, deltoïde antérieur gauche, deltoïde postérieur gauche, lombaire gauche. Pour deux novices, une activité musculaire bien supérieure à l'ensemble des 8 fossoyeurs est enregistrée pour le muscle deltoïde antérieur gauche et pour les muscles lombaires. Pour le deltoïde moyen gauche, la moyenne de l'EMG max est significativement plus élevée pour les expérimentés par rapport aux novices. 
Figure 3 : Moyenne (et écart type) de l'EMG maximal pour les fossoyeurs expérimentés (barres pleines) et les novices (barres vides) des muscles : deltoïde antérieur droit (DAD), deltoïde moyen droit (DLD), deltoïde postérieur gauche (DPD), lombaire droit (LD), deltoïde antérieur gauche (DAG), deltoïde latéral gauche (DLG), deltoïde postérieur gauche (DPG) et lombaire gauche (LG) ; ' $* \star^{\prime}$ = différence significative $(p<0.05)$.

Figure 3: Mean (standard deviation) of maximal EMG for experienced gravediggers (filled bars) and novices (empty bars) for different muscles: anterior right deltoid (DAD), middle right deltoid (DLD), posterior right deltoid (DPD), right lumbar (LD), anterior left deltoid (DAG), middle left deltoid (DLG), posterior left deltoid (DPG) and left lumbar (LG); ${ }^{\prime * *^{\prime}}=$ significant difference $(p<0.05)$

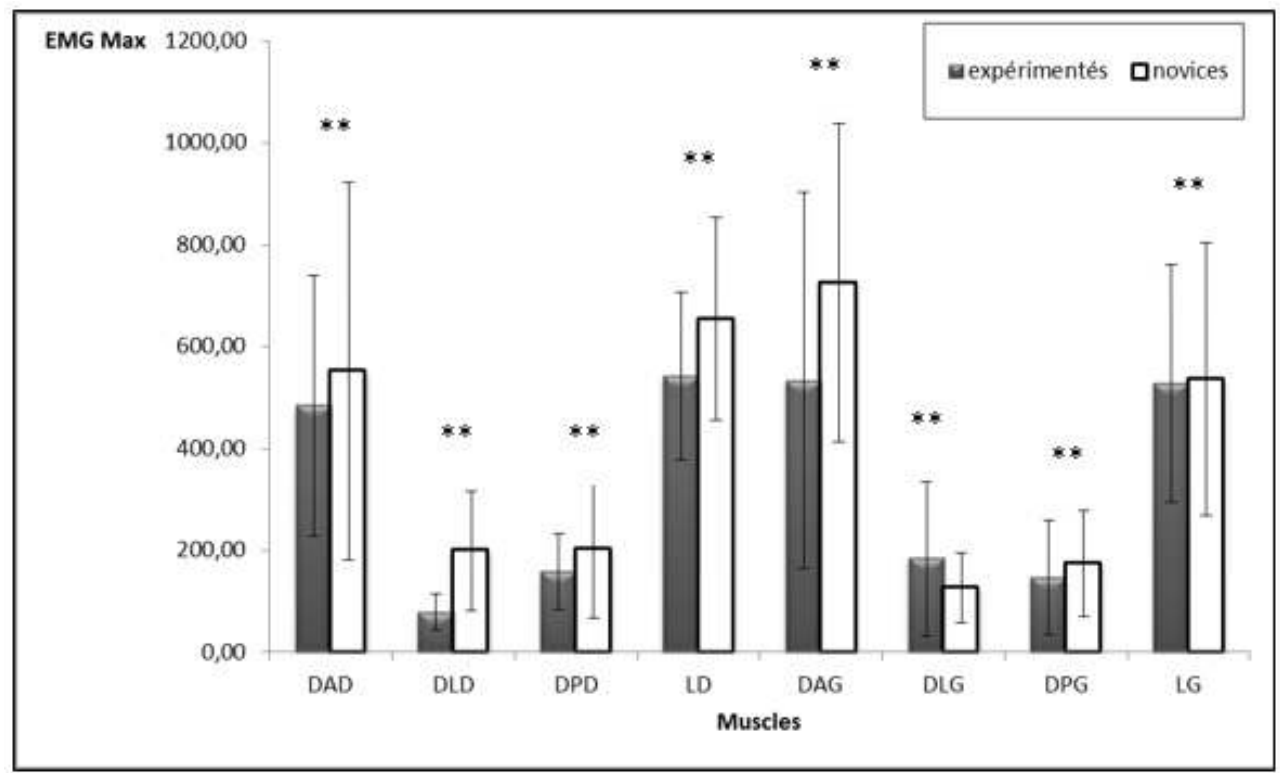

L'analyse de la variabilité intra-sujet, réalisée par régression en prenant en compte la moyenne de l'EMG max pour chaque muscle et pour chaque fossoyeur à chaque jeté arrière, montre des différences significatives (Tableau 3) pour divers muscles pour les novices et pour les expérimentés. Ces résultats montrent une variabilité de l'activité musculaire pour tous les fossoyeurs volontaires indépendamment de l'expérience. Néanmoins, il est à constater que cette variabilité est significative sur des muscles (et régions du corps) différents.

Tableau 3 : Analyse de régression prenant en compte l'EMG max de l'ensemble des cycles pour chaque muscle et chaque fossoyeur ; ${ }^{\prime \star \star}{ }^{\prime}=$ différence significative $(p<0.05){ }^{\prime}-{ }^{\prime}=$ différence non significative $(p>0.05)$.

Table 3: Regression analysis of maximum EMG for all cycle movements for each muscle and each gravedigger; ${ }^{\star \star \star}{ }^{\prime}=$ significant difference $(p<0.05) ;^{\prime \prime}{ }^{\prime}=$ non-significant difference $(p>0.05)$

\begin{tabular}{|c|c|c|c|c|c|c|c|c|c|}
\hline & \multirow[t]{2}{*}{ Expérience } & \multicolumn{8}{|c|}{ Muscle } \\
\hline & & DAD & DLD & DPD & LD & DAG & DLG & DPG & LG \\
\hline Fossoyeur 1 & expérimenté & - & - & $* *$ & $* *$ & - & - & $* *$ & - \\
\hline Fossoyeur 2 & novice & - & $* *$ & - & $* *$ & $* *$ & $* *$ & - & ** \\
\hline Fossoyeur 3 & expérimenté & ** & $* *$ & - & $* *$ & - & - & $* *$ & - \\
\hline Fossoyeur 4 & expérimenté & - & - & ** & - & - & $* *$ & - & - \\
\hline Fossoyeur 5 & novice & - & - & - & - & $* *$ & $* *$ & - & $* *$ \\
\hline Fossoyeur 6 & novice & $\neq 8$ & - & - & - & - & $* *$ & - & - \\
\hline Fossoyeur 7 & novice & $* *$ & $* *$ & $* *$ & $* *$ & $* *$ & ** & - & - \\
\hline Fossoyeur 8 & expérimenté & $\neq 8$ & - & ** & $* *$ & - & - & $* *$ & - \\
\hline
\end{tabular}

Prenant en compte la durée du cycle, les analyses montrent qu'il n'y a pas de différence significative entre le groupe des expérimentés et celui des novices (Figure 4). 
L'ensemble des fossoyeurs réalise les jetés arrière dans un temps compris entre $2 \mathrm{~s}$ et $3,5 \mathrm{~s}$ pour une durée moyenne de $2,5 \mathrm{~s}$.

Figure 4 : La moyenne (et l'écart type) de la durée du cycle pour le groupe des expérimentés (barres pleines) et des novices (barres vides).

Figure 4: Mean (standard deviation) of cycle for experienced gravediggers (filled bars) and novice gravediggers (empty bars)

\section{Durée cycle (s) Durée du cycle}

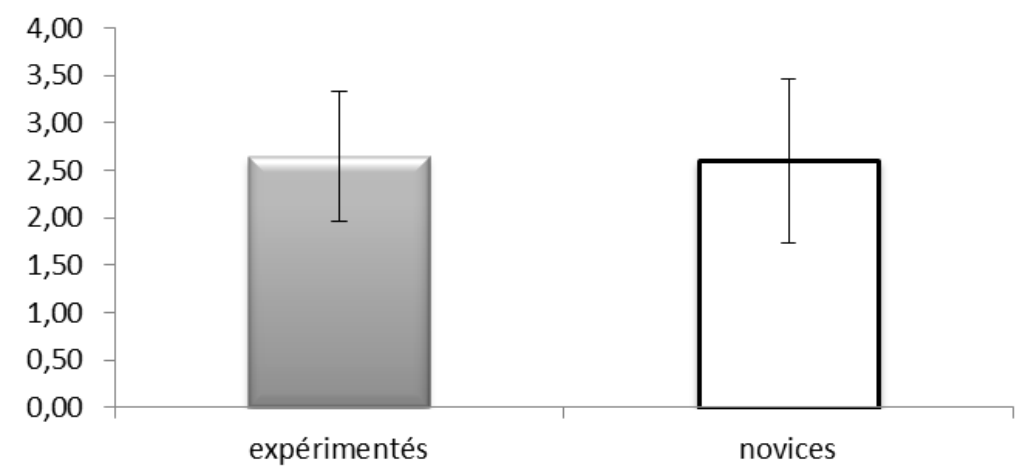

\subsection{Présentation des résultats des activités musculaires à l'ensemble des fossoyeurs volontaires}

Une fois le traitement des données des activités musculaires réalisé, les résultats de ces analyses sont présentés, par les biomécaniciens, de manière globale aux 8 fossoyeurs qui ont participé au recueil de données. Les objectifs de cette étape sont : d'apporter ces résultats à la connaissance des fossoyeurs et de préparer l'étape des autoconfrontations simples et croisées. Cette présentation était précédée d'un rappel du cadre dans lequel le recueil de données a été réalisé, de la méthodologie d'analyse et notamment de la définition du geste de jeté arrière (cf. § 2.3.1.4.).

\subsection{Organisation des auto-confrontations simples et croisées entre fossoyeurs participants sur la base des résultats de l'analyse des activités musculaires}

À l'étape de l'organisation des auto-confrontations, l'enjeu est de rendre accessible les résultats de l'analyse des activités musculaires au chercheur psychologue du travail et aux fossoyeurs. Ils doivent les utiliser comme outils d'analyse des gestes de jeté arrière dans les perspectives du cadre méthodologique présentées dans la partie 1 de l'article.

\subsubsection{Réalisation du support technique des auto-confrontations}

Dans cette optique, un outil est créé en vue d'être utilisé comme support des séances d'auto-confrontations. La représentation graphique des résultats associée à la vidéo a 
pour but d'engager les fossoyeurs à une observation minutieuse, image par image, des jetés arrière.

Ainsi, pour quatre zones anatomiques (épaule droite, épaule gauche, lombaire droit, lombaire gauche), deux gammes de gestes (extrait de l'ensemble des cycles enregistrés) sont définies à partir de l'amplitude des EMG: une gamme de gestes les moins sollicitants (au moins 5 pour chaque fossoyeur) et une gamme de gestes les plus sollicitants (au moins 5 pour chaque fossoyeur). Un montage vidéo de ces cycles est également réalisé. Sur ce montage vidéo chaque cycle est numéroté de façon à retrouver facilement si le geste est plus ou moins sollicitant (Figure 5). Ces éléments composent le support technique des auto-confrontations simples et croisées réalisées avec les fossoyeurs volontaires.

Figure 5 : Exemple de montage des résultats de l'analyse des activités musculaires (moyenne EMG) et de la vidéo assiociée des quatre zones anatomiques analysées (EP D : épaule droite, cercle bleu ; EP G : épaule gauche, cercle rouge ; Lomb D : lombaire droit, losange bleu ; Lomb $\mathrm{G}$ : lombaire gauche, losange rouge).

Figure 5: Example of presented results including muscular activity (mean EMG) and associated video recordings for different anatomical zones (EP D - red circles= right shoulder; EP G - blue circles= left shoulder; Lomb $D$ - blue rhomb= right lumbar; Lomb G - red rhomb= left lumbar)

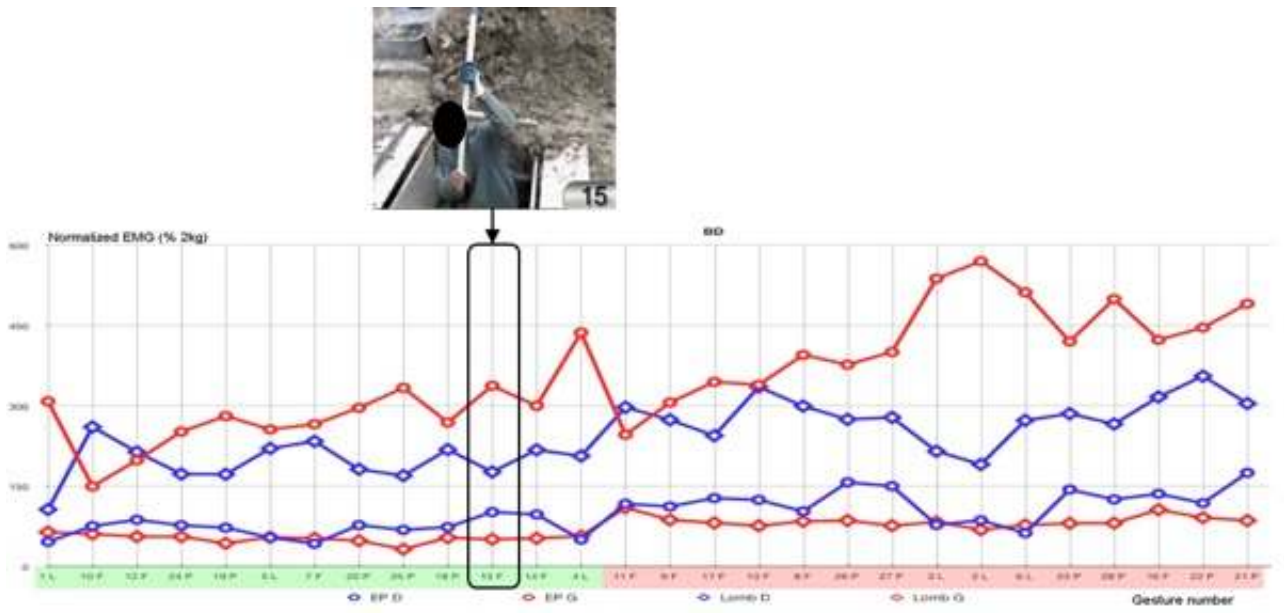

\subsubsection{Réalisation des auto-confrontations simples et croisées}

L'auto-confrontation simple a lieu en dehors du poste de travail et a pour objectif de placer chaque fossoyeur en situation d'analyser ses propres résultats, ses propres gestes. L'auto-confrontation simple est effectuée selon un schéma : sujet auto confronté / supports techniques mobilisés (ici montage vidéo et représentations graphiques) / intervenants chercheurs (clinicien de l'activité et biomécanicienne). La consigne donnée au fossoyeur est de commenter ce qu'il se voit faire ou ne pas faire sur la vidéo. Les commentaires du fossoyeur et les échanges avec l'intervenant sont enregistrés sur support vidéo. Dans le cadre de cette association avec les résultats des activités musculaires, l'auto-confrontation simple se déroule en deux temps : d'abord le sujet est confronté à son propre support technique (analyse EMG + vidéo) et ensuite au support technique de certains de ses collègues dans le but de préparer l'étape suivante de discussion entre collègues sur la base de leurs analyses respectives.

L'auto-confrontation croisée a lieu en dehors du poste de travail et selon un schéma: deux sujets auto-confrontés / supports techniques de chacun d'eux / intervenants 
chercheurs. La constitution des binômes de fossoyeurs auto-confrontés est réalisée de la façon suivante: plus les écarts des résultats des activités musculaires sont importants entre deux fossoyeurs et plus il est cherché par les intervenants, avec leur accord, de les réunir lors d'auto-confrontation croisée. La consigne donnée à chacun est de commenter, à tour de rôle, la vidéo du collègue présent, chacun d'eux ayant fait préalablement l'expérience de l'auto-confrontation simple. Les objectifs de cette étape sont : de provoquer entre fossoyeurs une discussion sur leurs différentes façons de faire le geste de jeté arrière, permettre à ce que l'expertise de chacun s'étoffe grâce aux échanges adossés à l'analyse des détails (y compris des activités musculaires) de leurs gestes les plus automatisés et invisibles à leurs propres yeux et aussi de conduire les fossoyeurs à redécouvrir leurs questions de métier et les réponses à y apporter au plan individuel, collectif et organisationnel. Les commentaires des sujets sont enregistrés sur un support vidéo en vue d'être exploités avec leur accord auprès de leurs collègues et des membres du comité de pilotage destinataires des questions soulevées par ces analyses.

\subsection{Analyse multimodale des auto-confrontations croisées}

41 Le court extrait suivant montre comment deux fossoyeurs (Figure 6) sont parvenus à s'approprier les résultats de l'analyse des activités musculaires, sous la forme du support technique, pour les utiliser comme moyen d'échanger sur leurs manières respectives d'exécuter le geste de jeté arrière. Cet extrait d'auto-confrontation croisée de deux fossoyeurs (DB et GD) et animée par l'intervenant chercheur en clinique de l'activité (In) permet de mieux rendre compte comment chacun cherche à résoudre les problèmes qu'il rencontre dans l'exécution de ce geste en s'essayant à la manière de l'exécuter par son collègue.

Figure 6 : Fossoyeurs GD et DB montrant le geste de jeté arrière au collègue lors d'une autoconfrontation croisée.

Figure 6: Gravediggers $G D$ and BD showing backward throws during crossed confrontations

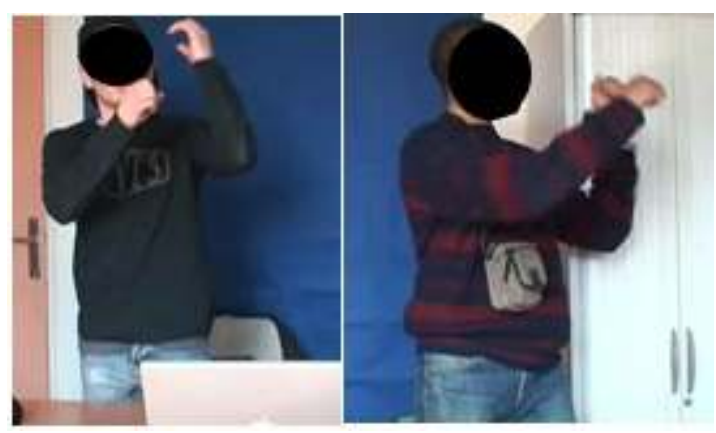


Figure 7 : Extrait d'auto-confrontation croisée. Figure 7: Cross-self-confrontation (Extracts)

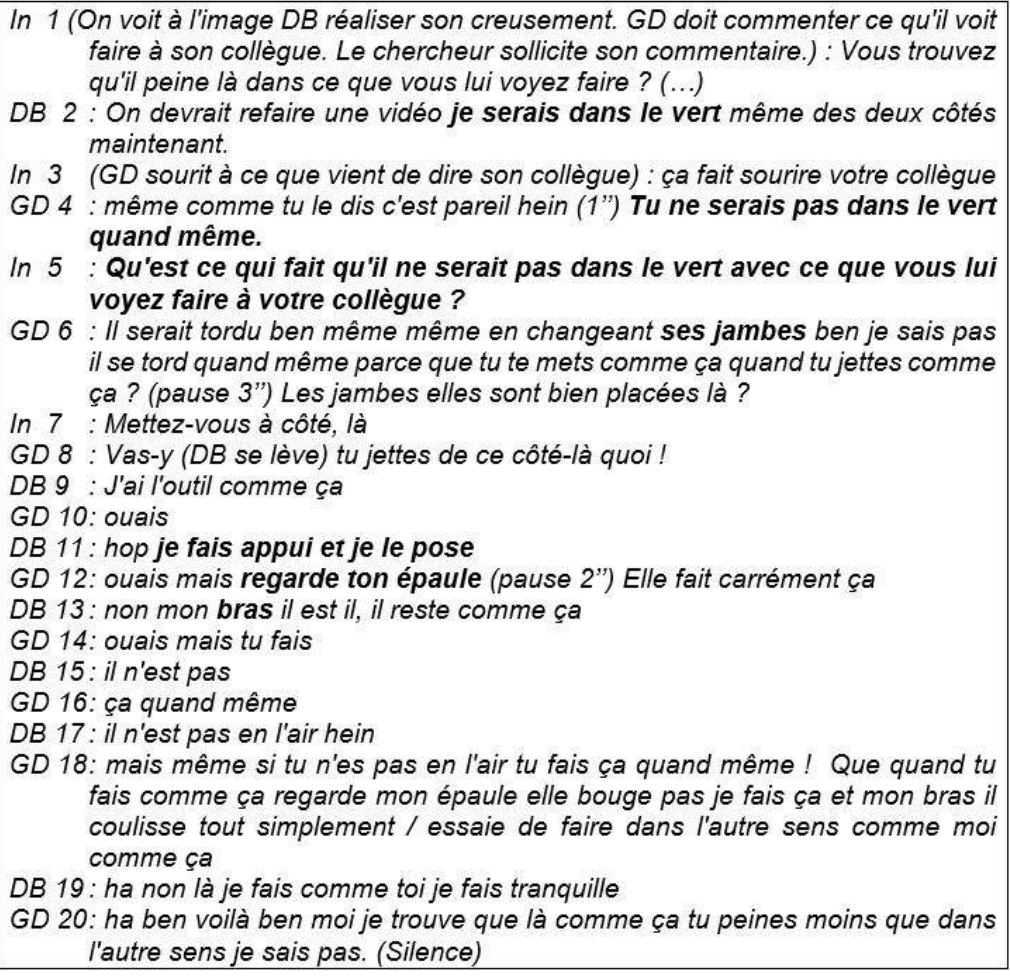

42 En caractère gras dans cet extrait (Figure 7) sont soulignées des expressions qui montrent comment les fossoyeurs et l'intervenant chercheur utilisent le support technique tiré des analyses des activités musculaires pour stimuler leur dialogue professionnel. Tous les fossoyeurs confrontés aux éléments du support technique (la vidéo et les résultats électromyographiques catégorisant les jetés arrière les plus et les moins sollicitants) mobilisent à des degrés divers, leur corps dans le dialogue par des simulations répétées du geste étudié en passant par l'imitation du geste du collègue (Simonet, 2011). La production de ces réalisations corporelles dans un dialogue tonique entre eux (Ajuriaguerra, 1962 ; Bullinger, 2004) prend même parfois le dessus sur la production d'énoncés langagiers: on peut notamment le noter quand les mots se révèlent des outils trop limités pour comprendre ces dilemmes «tonico-posturaux » de l'activité de creusement et ces questions de placement du corps dans la fosse que chacun d'eux cherche à mieux saisir dans le débat.

Ces résultats de l'action nous conduisent à proposer une analyse multimodale, en nous rapprochant des travaux de Cosnier (Cosnier, 1996, 1997), pour la transcription des énoncés argumentatifs verbaux et gestuels et à la technique de notation Benesh (Mirzabekianz, 2000) pour la notation du geste réalisé au cours du dialogue. La nature de l'appropriation par les fossoyeurs des résultats issus de l'analyse des activités musculaires et des images vidéos nous invite à interroger les sensations que chacun d'eux cherche à expérimenter. C'est la raison pour laquelle nous proposons une analyse multimodale des traces de cette activité d'analyse en l'inscrivant dans l'examen d'un rapport entre énoncés langagiers et expériences sensibles. Cette dernière dimension peut être révélée à l'intervenant à partir d'une technique de notation qui lui permet de pointer que les déplacements de quelques centimètres du bras, du coude, de l'angle ouvert de l'épaule ou de tout autres déplacements des segments du corps engagés dans 
le geste imité, refait à blanc, ouvrent le sujet sur l'expérimentation de nouvelles sensations. De nouvelles sensations qui permettent aussi de s'imaginer procéder un peu autrement ou d'affirmer sa manière habituelle de s'y prendre en s'essayant aux voies de réalisations empruntées par d'autres dans le métier. La notation du continuum des reprises du geste au cours de l'auto-confrontation nous donne accès à l'expérience sensible à laquelle le fossoyeur se livre quand il reprend son geste au contact de celui de son collègue. La notation donne aussi accès, pour partie, à la richesse des différentes gammes expérimentées et ressenties par le fossoyeur d'une répétition à l'autre. C'est alors l'engagement du corps au travail qui fait l'objet de l'expérimentation à laquelle se livrent les fossoyeurs dans un dialogue tonique stimulé par des auto-confrontations croisées organisées sur la base des analyses biomécaniques. Une dynamique qui peut permettre la fabrication d'un geste dialogué favorable à la prévention des TMS (Simonet \& Caroly, 2020).

Finalement, chacun tente de trouver dans des exécutions éloignées du style qui caractérise sa manière habituelle de réaliser son jeté arrière le moyen de dialoguer avec sa problématique professionnelle personnelle en passant par celle des autres. Par exemple, alors que le jeté arrière de GD en situation réelle de travail se caractérise par un jeté arrière coulissé du manche de l'outil dans sa main "spécialisé côté gauche " (jetant la terre par-dessus l'épaule gauche) et un corps penché côté opposé (contrairement d'ailleurs à ce qu'il en dit en GD25), ce dernier, confronté à la manière de s'y prendre de son collègue, répète des jetés arrière alternés en prenant soin de «rester droit ». Dans ce même temps d'échange, DB dont le style de jeté arrière en situation réelle de travail se caractérise par une alternance de jetés arrière non coulissés s'essaye lors de l'auto-confrontation croisée aux jetés arrière en faisant coulisser le manche de son outil entre ses mains.

L'usage fait par chacun de son corps dans l'activité ordinaire de travail et les simulations d'autres manières de s'y prendre par voie d'imitation à celle du collègue, nous permet d'avancer que dans ces expérimentations de jetés arrière plus variés qui font sens pour chacun d'eux, chacun réévalue sa gamme opératoire et sensori-motrice. Cela peut lui permettre de repenser l'engagement de son corps dans l'activité, plus globale, de creusement. Cet "élargissement du champ des actions [...] caractéristique typique et fondamentale du développement humain" (Wisner, 1997, p. 250) synonyme de développement du pouvoir d'agir (Clot, 2008) comporte des potentialités nouvelles d'exécution des gestes. Il va s'en dire qu'elles seront plus ou moins réalisables au regard des caractéristiques variables de la fosse à creuser d'une fois sur l'autre et de celles des fossoyeurs et de l'organisation du travail collectif des fossoyeurs.

\section{Résultats de l'action interdisciplinaire}

Nous avons détaillé dans les parties précédentes les méthodes et outils mobilisés pour expliquer comment le geste étudié à la demande des fossoyeurs et du médecin du travail est réalisé, enregistré, recomposé et défini par les deux équipes de chercheurs pour analyser les sollicitations musculaires puis outiller les auto-confrontations simples et croisées des fossoyeurs. Dans cette partie, nous insisterons davantage sur le statut de l'analyse des activités musculaires comme instrument du développement de leur geste de métier dans l'activité des fossoyeurs. 
Figure 8 : Migration fonctionnelle du geste. Figure 8: Functional migration of the gesture

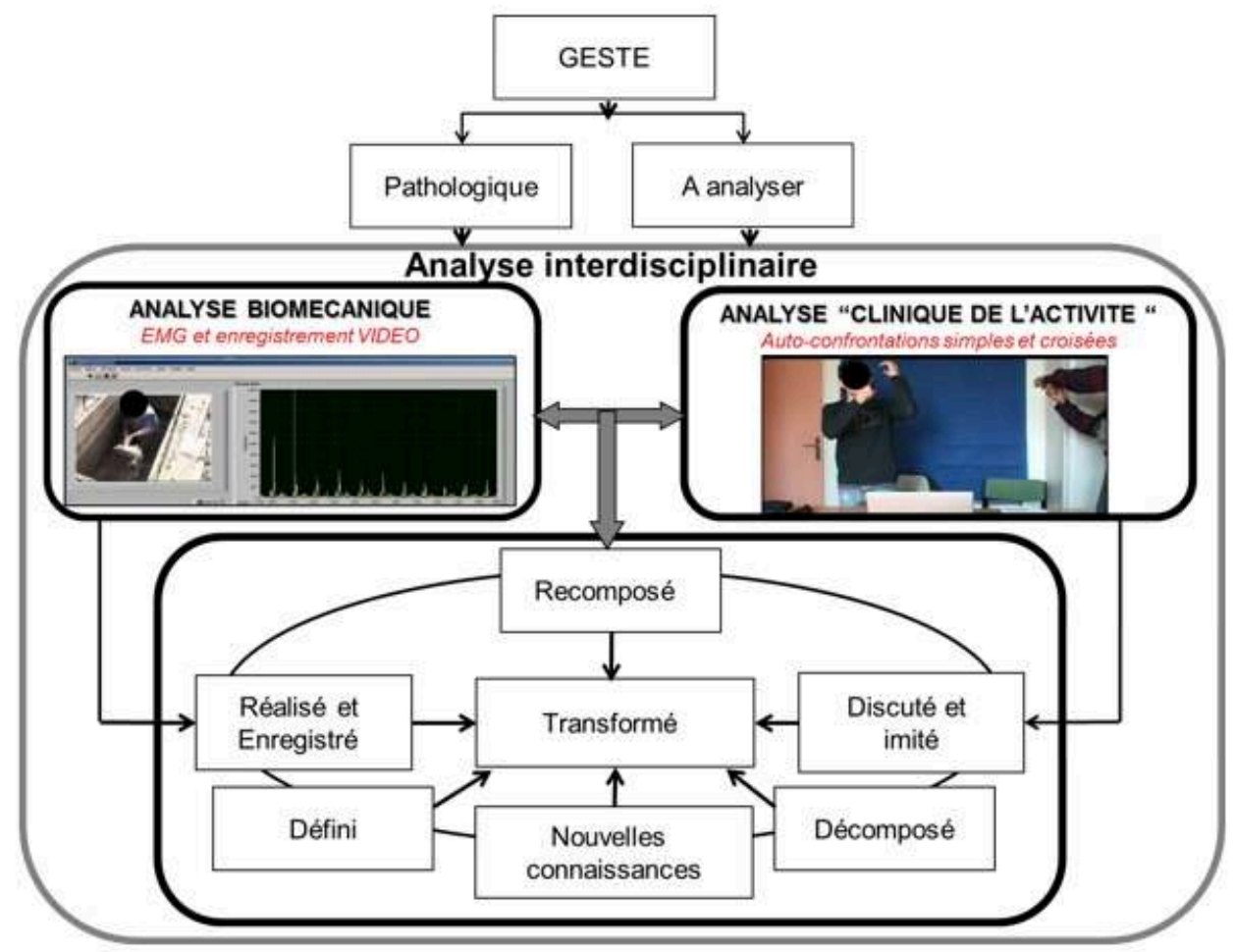

Cette figure montre que l'analyse mettant en œuvre la méthodologie interdisciplinaire permet aux fossoyeurs d'examiner à nouveau et dans un autre contexte, différent de celui de l'activité réelle et incluant l'analyse du mouvement (au travers des activités musculaires), les critères de réalisation d'un geste particulièrement délicat à effectuer, voir qui peut être source de pathologie. Ainsi, à travers le support technique, comprenant des résultats de l'analyse des activités musculaires et l'enregistrement vidéo synchrone et utilisé lors des auto-confrontations simples et/ou croisées, le geste de jeté arrière devient un objet de discussion plus riche des variantes stylistiques discutées en passant d'un contexte à l'autre de sa réalisation (activité concrète observée, 1ères séries d'auto-confrontations, restitutions, mesures des activités musculaires, nouvelles séries d'auto-confrontations à l'aide d'un support technique construit sur la base des résultats des activités musculaires). C'est dans la traversée de ces contextes que ce geste devient plus disponible pour la discussion professionnelle et in fine plus disponible, pour chacun, comme instrument de développement de son activité professionnelle. Ainsi ce geste de jeté arrière passe du statut de geste à risque et difficile, que certains fossoyeurs ne veulent pas faire alors même qu'il est incontournable dans une fosse entre 1,50 et $2 \mathrm{~m}$ de profondeur, au statut de geste à étudier de manière détaillée, de geste réalisé et enregistré, de geste défini par les chercheurs, de geste discuté lors des auto-confrontations simples et croisées, de geste décomposé en plusieurs éléments, de geste enrichi par de nouvelles connaissances et finalement au statut d'un geste dialogué (Simonet \& Caroly) permettant à chaque fossoyeur de s'imaginer faire un peu autrement en situation réelle de travail (Figure 8). Cette circulation du geste entre ces différents contextes lui donne un statut différent d'un contexte à l'autre : elle permet de délier, en passant par l'analyse du mouvement, le geste étudié de «l'identité » du fossoyeur (être du métier) qui l'exécute pour l'y 
relier, ensuite et autrement, après le débat dont il a fait l'objet et dans lequel le fossoyeur l'a refait pour lui-même au contact des autres (faire son métier). L'association interdisciplinaire entre analyse des activités musculaires et clinique de l'activité permet d'ouvrir plus largement la zone de développement potentiel de la formation du geste en stimulant leurs échanges sur les questions de métier qui s'y rattachent.

Dans la traversée de ces contextes où le geste étudié est redéfini, discuté, imité, etc., on peut faire apparaître que :

- le dialogue se développe dans une alternance d'énoncés langagiers et de réalisations gestuelles qui organise l'activité argumentative et stimule la réflexion des fossoyeurs lors des auto-confrontations. Dans cette alternance, l'examen du geste empêché de l'un devient le moyen de repousser les propres limites du geste de l'autre. En effet, chacun enrichit son expérience corporelle en s'expliquant avec le geste des autres : positions et engagement du corps dans la fosse pour le creusement ;

- le travail du corps dans l'activité est stimulé par la confrontation quand des styles individuels marqués par leurs différences trouvent à se discuter au contact des règles transpersonnelles ou génériques du métier (cf. : note 1);

- au-delà du geste de jeté arrière lui-même, cette construction méthodologique qui fait circuler le geste dans ses différents registres impersonnel, personnel, interpersonnel et transpersonnel du métier (cf. : note 1) contribue à stimuler des interrogations sur d'autres dimensions organisationnelles du métier de fossoyeur : le matériel utilisé, l'organisation du travail dans les équipes, la formation des novices et des experts... Ces réflexions permettent de « faire vivre » les résultats présentés ici pour les intégrer dans une perspective globale de prévention des TMS en comité de pilotage et au CHSCT (Massot \& Simonet, 2017 ; Poussin \& Simonet, 2017).

\section{Discussion}

\subsection{Le statut des résultats de l'analyse biomécanique dans la méthodologie clinique de l'activité}

$\mathrm{Au}$ plan de la visée méthodologique clinique de l'activité qui est celle du développement du pouvoir d'agir des professionnels et des marges de manœuvres individuelles, collectives et organisationnelles (Clot \& Simonet, 2015) et dans le cadre d'une intervention centrée sur la prévention des TMS, cette association interdisciplinaire avec la biomécanique permet d'enrichir la méthode des autoconfrontations simple et croisée traditionnellement mobilisée en clinique de l'activité (Clot, Faïta, Fernandez, \& Scheller, 2000).

Sur un plan plus théorique, la visée de la clinique de l'activité est le développement de l'activité. La visée de la biomécanique est davantage de mieux comprendre le fonctionnement de l'appareil moteur et dans ce cas des membres supérieurs et du tronc. L'interdisciplinarité consisterait dans ce contexte à faire en sorte qu'en clinique de l'activité nous recentrions notre attention sur le fonctionnement du développement de l'activité en même temps que les biomécaniciens porteraient attention aux fonctionnements de l'appareil moteur dans une optique de développement. 
51 L'introduction d'outils tirés de l'analyse biomécanique (composantes du support technique des auto-confrontations) permet au cadre clinique de l'activité :

- de provoquer une dynamique psychosociale de «double stimulation» de reprise d'un énoncé dans un autre énoncé et, alternativement, de simulation d'un mouvement corporel dans un autre mouvement corporel (Vygotsky, 1997)

- de conduire une analyse multimodale des invariants du développement des gestes dans l'activité

- de permettre aux fossoyeurs des avancées significatives sur leurs questions de métier qui n'ont pas trouvé à se discuter avec autant de précisions et de détails lors des précédentes auto-confrontations réalisées sans association avec l'analyse de l'activité musculaire.

Les supports techniques tirés de l'association avec l'analyse biomécanique provoquent une «double stimulation » de la pensée et de l'action (Vygotski, 1978) dans ce cadre méthodologique des auto-confrontations. Comme cela a déjà été démontré par d'autres travaux inscrits dans la perspective de développement de l'activité (Engeström \& Sannino, 2013) et par d'autres travaux en clinique de l'activité ouverts à l'interdisciplinarité (Fernandez, 2015 ; Kloetzer, Quillerou-Grivot, \& Simonet, 2015), le contexte expérimental a pour finalité de devenir un contexte d'expérimentation dans lequel l'intervenant produit ses propres outils (films des jetés arrières, graphiques des jetés arrières les plus et les moins sollicitants) pour installer un cadre d'analyse de l'activité (ici par auto-confrontations) favorable à la construction par les sujets de nouveaux énoncés et de nouvelles habiletés psychomotrices. Cette visée développementale pose des problèmes techniques aux intervenants, car les professionnels doivent s'approprier leurs outils (appelés artefacts en clinique de l'activité) pour pouvoir les utiliser comme instruments techniques et psychologiques de leur propre production. Ces questions ne sont pas nouvelles. Vygotski (Vygotski, 1927/1999) en son temps a critiqué la mesure psychométrique quand elle se limite à mesurer le niveau de développement psychologique atteint du sujet mesuré : en le réduisant à son fonctionnement actuel, elle écarte toutes perspectives développementales. En portant cette critique, Vygotski ne rejette pas de manière doctrinaire l'expérimentalisme et les méthodes quantitativistes. Ce point est capital pour bien comprendre le sens de notre action interdisciplinaire entre biomécanique et clinique de l'activité. Notre volonté est de dépasser le clivage stérile entre méthodes qualitatives et méthodes quantitatives, tant les unes peuvent être ressources pour les autres quand cela s'avère nécessaire à l'avancement de la recherche et de l'action et quand les écarts entre les approches parviennent à être travaillés (Savescu \& Simonet, 2020). À partir d'expériences cliniques où il met le sujet en situation de se mesurer à un protocole expérimental dans une perspective de déploiement des forces de compensation réussie de la maladie, Vygotski cherche à faire des protocoles expérimentaux des instruments techniques du développement de l'activité du sujet (Vygotski, 1978). Notre action interdisciplinaire s'inscrit dans cette perspective-là de l'organisation des conditions du développement de l'activité et du geste de métier en activité. Mais cette perspective a ses exigences. Aussi les intervenants-chercheurs biomécaniciens et de la clinique de l'activité ont-ils dû consentir aux efforts réciproques nécessaires pour s'engager au-delà de l'addition de compétences entre plusieurs disciplines très éloignées l'une de l'autre qui caractérise l'action pluridisciplinaire pour aller vers une action interdisciplinaire qui «suppose un dialogue, un échange ou une confrontation entre plusieurs disciplines ». Il ne s'agit pas d'une simple juxtaposition, mais d'une interaction et d'une interpénétration (Savescu \& 
Simonet, 2020). Les disciplines en ressortent alors transformées, même si «les changements ne sont souvent que périphériques » (Vinck, 2002).

Aussi certains des effets sur le cadre méthodologique clinique de l'activité du recours à l'analyse biomécanique peuvent être présentés :

- Engagement du corps par voie de simulations et d'imitations des alternatives d'effectuation du geste objet de débat dans le genre professionnel. L'activité pratique n'est pas dissociable d'une activité sur soi en ce sens que «le geste modifie, en même temps que le milieu, celui qui le fait. »(Wallon, 1942/1970, p. 194).

- Production d'artefacts comme la création d'un geste étalon de mesures biomécaniques qui est nécessaire à l'opération de décontextualisation-recontextualisation du geste dans l'activité ${ }^{7}$ il n'existe pas de geste sans action réalisant une activité concrète (le creusement d'une fosse), car le geste réalise l'action, mais la séparation du geste de l'action première dans le cadre d'une autre activité (celle de l'analyse par auto-confrontations) est une opération nécessaire quand on veut offrir aux sujets l'occasion de le refaire en revisitant les voies possibles de transformation de leur engagement psychomoteur et psychosocial dans le métier. Ces sensations kinesthésiques nouvellement expérimentées ne trouvent pas de mots pour les décrire. C'est une partie du geste qui échappe au langage (notamment l'activité musculaire), car toute une partie du geste n'est pas consciente (Fernandez, 2015). Dans l'énergie déployée par les sujets engagés dans ces cycles de répétitions de leurs gestes qui luttent contre leurs répétitions ordinaires au travail, ces derniers transforment leur rapport à l'efficience des techniques corporelles et au sens de l'engagement de leur corps dans l'activité de travail (Tomás, Simonet, Clot, \& Fernandez, 2009).

\subsection{Le statut de la méthodologie clinique de l'activité pour les résultats de l'analyse biomécanique}

En tant que science expérimentale, la biomécanique apporte des éléments de plausibilité biologique ou dans le cadre de l'étude ergonomique pilotée par l'ergonome, fournit des informations souvent métrologiques, utiles à la compréhension ou à la transformation du travail (Aptel \& Vézina, 2008). Dans leur approche combinée Plamondon \& Denis (Plamondon \& Denis, 2008) ont souligné que si les observations ergonomiques permettent d'identifier les éléments qui apparaissent le mieux contribuer à l'efficience du geste, l'approche biomécanique aide à quantifier les effets sur le chargement des tissus et le travail mécanique résultant (le déplacement des segments corporels). Dans une autre étude d'exploitation de la variabilité gestuelle pour la prévention des TMS (Brunet \& Riff, 2009), les auteurs évoquent des difficultés rencontrées pour ce type d'analyse lors de différentes méthodologies telles que :

- l'interruption de l'opérateur dans son travail pour engager une discussion sur le geste est difficile pour des raisons culturelles, leur surprise ou leur difficulté à parler du « faire ",

- la lourdeur et le temps consacré à ce type d'analyse faisant appel à des techniques d'observation systématique (220 observations) ou la réalisation des traces de l'activité pour des entretiens en auto-confrontation (basés sur 180 enregistrements vidéo de l'activité des opérateurs).

Du point de vue de ces difficultés, l'analyse biomécanique facilite l'identification de différentes façons de réaliser le même mouvement.

Par ailleurs, dans cette étude, prenant en compte des variables validées par la littérature (Madeleine \& Madsen, 2009; Madeleine, Voigt, \& Mathiassen, 2008; 
Mathiassen, Möller, \& Forsman, 2003), la méthodologie biomécanique se heurte surtout aux exigences d'enregistrement (en plein air et dans une fosse entre $1 \mathrm{~m} 50$ et $2 \mathrm{~m}$ de profondeur) et de forme de présentation des résultats pour la réalisation du support technique (cf. § 2.5.1.) utilisé lors des auto-confrontations simples et croisées dans le cadre d'une méthodologie clinique de l'activité. En effet, les résultats biomécaniques doivent participer à ouvrir et à encourager le dialogue entre professionnels, et non à le fermer. L'analyse du mouvement, à l'aide de l'électromyographie, aurait pu s'arrêter à porter à la connaissance des fossoyeurs et de leur hiérarchie des variabilités inter et intra-individuelles mesurées. Mais dans cette action interdisciplinaire, les résultats biomécaniques dépassent le statut d'apport de connaissances sur le mouvement pour devenir objet de discussion entre professionnels afin de leur permettre de "voir " autrement leur geste et ainsi le développer. Mais cette évolution de statut n'est pas sans poser de difficultés, car il est connu que lors des interventions ergonomiques participatives (Van Eerd, Cole, Irvin, Mahood, Keown, Rheberge et al., 2010), l'historique de l'intervention peut faciliter ou inhiber le déroulement de l'action actuelle. En effet, les fossoyeurs, qui ont déjà participé à l'analyse de leur activité, auraient pu prendre les résultats de l'analyse biomécanique comme une vérité absolue sans pouvoir ou vouloir la discuter. Dans cette action, le challenge de la biomécanique est de tenir les deux registres : l'exactitude des résultats et la possibilité de les discuter par les fossoyeurs à travers le support technique réalisé. Ainsi, les résultats présentés soutiennent les efforts des fossoyeurs dans un processus de décomposition et recomposition du geste, en passant par l'analyse du mouvement, au sein des collectifs de travail. La variabilité est donc conçue comme un opérateur de transformation et de santé quand elle est discutée entre experts dans le métier. C'est le développement de la variabilité propre qui est visé dans cette dynamique collective. Ainsi, les dissonances produites par l'examen méthodique de la variabilité intra et interindividuelle alimentent pour chacun, quand elles sont instruites dans l'échange entre pairs, le dialogue et la controverse professionnelle comme ressource du collectif de travail. La variabilité des mouvements et postures des fossoyeurs habituellement examinée du côté des risques à régler par le préventeur finit ici par être envisagée comme moyen de développer de nouvelles ressources pour l'action des fossoyeurs et leur santé. Au début de l'intervention, c'est le discours de la fatalité qui domine : " chacun fait à sa manière et toutes les manières se valent, il est donc inutile d'en parler ", « de toute façon se faire mal au dos c'est normal quand on est fossoyeur ", "on fait la même chose ». Cette expression de "tout le monde travaille pareil ", déjà évoquée dans la littérature (Brunet \& Riff, 2009), cristallise le sentiment de fatalisme face aux TMS. Dans ces conditions, est-ce que la variabilité peut servir à autre chose qu'à alimenter le fatalisme du côté des fossoyeurs et le désir managérial vain de la faire taire au profit de soi-disant bonnes pratiques dispensées par des formations gestes et postures éloignées des dilemmes du métier (Simonet, 2012) ? En alimentant les échanges entre fossoyeurs, les analyses biomécaniques, par l'autorité acquise qu'elles ont chez les personnels chargés de la prévention des risques, contribuent à installer dans l'organisation du travail les questions de métier à résoudre et la nécessité de nouvelles actions de prévention pensées et réalisées avec le concours des professionnels de terrain (Massot \& Simonet, 2017). La biomécanique ainsi utilisée permet d'enrichir les connaissances sur le mouvement et donc de surcroît sur le geste étudié, de redynamiser le collectif de travail des fossoyeurs et de donner aux membres du comité de pilotage de nouvelles connaissances sur la situation concrète de travail des fossoyeurs et de nouveaux motifs 
d'actions qui leur permettent de repenser la politique de formation du geste de métier, retouchant ainsi leur politique de prévention des TMS (Poussin \& Simonet, 2017; Simonet \& Poussin, 2014). Les résultats de cette étude montrent que l'analyse biomécanique, notamment utilisée dans des démarches de prévention de TMS qui se concentrent sur la réduction des risques (Thibault et al., 2013), peut aussi intervenir dans des démarches développementales par la mise en évidence et l'utilisation de la variabilité gestuelle.

\subsection{Méthodologie d'action interdisciplinaire pour la prévention durable des TMS}

57 Cette action interdisciplinaire permet de provoquer des conditions favorables à une circularité entre la pensée, le langage et le geste, par la création de contextes d'énonciation et de comparaison des variabilités gestuelles inter et intra-individuelles. Elle revitalise la fonction du collectif comme ressort du développement du geste de métier en nourrissant la comparaison aux autres et à ses autres possibilités de faire et d'agir. Elle permet aux professionnels de revisiter les situations de travail les plus concrètes et, ainsi, d'en redécouvrir toute l'épaisseur qui va des impasses aux possibilités non encore exploitées ou envisagées. Ainsi, chacun d'eux peut percevoir, mais aussi s'essayer autrement au geste de jeté arrière en réinterrogeant l'engagement de soi dans cette activité de creusement d'une fosse. Comme il a été évoqué dans l'extrait d'auto-confrontation croisée entre deux fossoyeurs, la répétition de la simulation gestuelle de l'un s'organise dans la reprise de la simulation gestuelle de l'autre. Le collectif est alors à envisager ici comme une instance de délibération en chacun des participants à l'action. Dans ce cas, les fossoyeurs participent aussi bien à la construction d'un contenu de formation comme d'autres ont pu l'envisager (Vézina, Prevost, Lajoie, \& Beauchamp, 1999) comme au développement de leurs propres gestes de métier (Clot, Fernandez, \& Scheller, 2007).

Les résultats issus de cette action interdisciplinaire doivent selon nous permettre aux préventeurs des TMS de développer des dispositifs de prévention à partir des buts qu'ils cherchent à atteindre. Ils permettent de repenser les dispositifs de formation du geste de métier en dépassant la barrière de la fatalité « on est fossoyeur donc on a mal au dos» ou encore la formation "aux bonnes pratiques». D'autres études pluridisciplinaires (Plamondon \& Denis, 2008) ont mis en avant le manque de convergence entre les techniques dites « sécuritaires » enseignées dans les programmes de formation et celles observées sur le terrain. Ces auteurs ont pris le pari de prendre en compte à la fois les considérations biomécaniques et les considérations de qualité du travail et de satisfaction que les professionnels retirent de leur travail. Les résultats de cette étude s'associent à ce type de démarche et montrent que les démarches de prévention existantes (Denis et al., 2005) peuvent s'enrichir et doivent offrir des espaces-temps d'élaboration continuée du geste dans le débat construit entre pairs et au-delà avec les autres acteurs de la prévention et les décideurs de l'organisation du travail. Ainsi, au plan méthodologique, les dispositifs de prévention doivent veiller à organiser ces possibilités pour les professionnels de comparer leurs gestes de métier dans le dialogue entre eux puis avec leur hiérarchie et les préventeurs. D'où l'intérêt de ne pas confondre entre des dispositifs qui ouvrent sur l'explicitation du geste déjà fait 
et ceux qui ouvrent sur l'expérimentation du geste possible à faire, tout en interrogeant les conditions de travail dans lesquelles il est possible de faire autrement.

Les résultats de cette action établissent un pont entre les opérateurs et les décideurs de l'organisation du travail quand le geste de métier devient un objet de discussion à partir de points de vue différents. Ils s'inscrivent dans une dynamique instaurée dans le collectif de travail pour une prévention durable de TMS tels qu'évoqués par Aptel et Vézina (Aptel \& Vézina, 2008).

\section{Conclusion}

Les résultats présentés montrent que la méthodologie interdisciplinaire permet d'ouvrir plus largement la zone de développement potentiel du geste dans l'activité des fossoyeurs en favorisant leurs discussions professionnelles. De même, l'analyse biomécanique n'a pas été exclusivement effectuée dans une optique d'apport de connaissances nouvelles. La mise en visibilité et en discussion dans le métier de la variabilité inter et intra-individuelle a ouvert le milieu professionnel (hiérarchie et CHSCT compris) (Massot \& Simonet, 2017) vers de nouvelles formes d'actions de prévention des TMS. La méthodologie clinique de l'activité mobilisée ici a bénéficié des apports de l'analyse biomécanique du geste pour développer la puissance d'action de son cadre méthodologique avec les collectifs de pairs, mais aussi avec les décideurs. Comme nous y encourage Jacques Leplat (Leplat, 2005, 2013), il conviendrait en analyse du travail d'accorder plus systématiquement toute sa place au geste de métier, à sa formation et à son développement. La prévention des TMS doit aussi pouvoir offrir des possibilités de reprises du geste propre en l'élevant au statut d'un geste dialogué ouvert sur de nouveaux contextes de sa réalisation tant dans sa formation que dans son exécution. Cette perspective interroge les outils d'action et la visée méthodologique des actions de prévention dans les milieux professionnels. Elle ouvre sur la question potentiellement jamais épuisée et méthodologiquement féconde des méthodes d'actions interdisciplinaires au service de l'implication active des professionnels et autres parties prenantes du milieu dans la prévention durable des TMS.

\section{BIBLIOGRAPHIE}

Ajuriaguerra, J. (1962). Le corps comme relation. Revue suisse de psychologie pure et appliquée, 21, 137-157.

Aptel, M., Cail, F., \& Aublet-Cuvelier, A. (2014). Les troubles musculosquelettiques du membre supérieur. Guide pour les préventeurs. INRS, $94 \mathrm{p}$.

Aptel, M., \& Vézina, N. (2008). Quels modèles pour comprendre et prévenir les TMS ? Pour une approche holistique et dynamique. Paper presented at the $2^{\mathrm{e}}$ Congrès francophone de recherche sur les TMS, Montréal, Canada. 
Boettcher, C. E., Ginn, K. A., \& Cathers, I. (2008). Standard maximum isometric voluntary contraction tests for normalizing shoulder muscle EMG. Journal of Orthopaedic Research, December, 1591-1597.

Bourgeois, F., \& Hubault, F. (2005). Prévenir les TMS. De la biomécanique à la revalorisation du travail, l'analyse du geste dans toutes ses dimensions. Activités, 2(1), 20-36. Retrieved from https://journals.openedition.org/activites/1561

Brunet, M., \& Riff, J. (2009). Analyse et exploitation de la variabilité gestuelle dans la prévention des TMS. PISTES, 11.

Bullinger, A. (2004). Le développement sensori-moteur de l'enfant et ses avatars. Un parcours de recherche. Ramonville Saint-Agne : Ères

Burden, A. (2010). How should we normalize electromyograms obtained from healthy participants? What we have learned from over 25 years of research. [https://doi.org/10.1016/ j.jelekin.2010.07.004]. Journal of Electromyography and Kinesiology, 20(6), 1023-1035.

Caroly, S., Coutarel, F., Escriva, E., Roquelaure, Y., Schweitzer, J. M., \& Daniellou, F. (2008). La prévention durable des TMS. Quels freins ? Quels leviers d'action? In F. Daniellou (Ed.), Rapport de recherche, PACTE, ANACT, LEEST, Équipe d'Ergonomie Bordeaux.

Clot, Y. (2008). Travail et pouvoir d'agir. Paris, France : PUF.

Clot, Y., Faïta, D., Fernandez, G., \& Scheller, L. (2000). Entretiens en autoconfrontation croisée : une méthode en clinique de l'activité. . PISTES, 2(1). Retrieved from www.pistes.uqam.ca/v2n1/ pdf/v2n1a3.pdf

Clot, Y., \& Fernandez, G. (2005). Analyse psychologique du mouvement : apport à la compréhension des TMS. Activités, 2(2), 68-78. Retrieved from https://journals.openedition.org/ activites/1818

Clot, Y., Fernandez, G., \& Scheller, L. (2007). Le geste de métier : problèmes de la transmission. Psychologie de l'interaction, 23-24, 109-138.

Clot, Y., \& Simonet, P. (2015). Pouvoirs d'agir et marges de manœuvre. Le Travail Humain, 78(1), 31-52. https://doi.org/10.3917/th.781.0031

Cosnier, J. (1996). Les gestes du dialogue. Psychologie de la motivation, 21, 129-138.

Cosnier, J. (1997). Sémiotique des gestes communicatifs. Nouveaux actes sémiotiques, 52(7-28).

Denis, D., St. Vincent, M., Jetté, C., Nastasia, I., \& Imbeau, D. (2005). Les pratiques d'intervention portant sur la prévention des troubles musculosquelettiques : un bilan critique de la littérature. IRSST.

Engeström, Y., \& Sannino, A. (2013). La volition et l'agentivité transformatrice : perspective théorique de l'activité Revue internationale du CRIRES : innover dans la tradition de Vygotsky, 1, 4-19.

Fernandez, G. (2004). Développement d'un geste technique. Histoire du freinage en Gare du Nord. PhD, CNAM, Paris.

Fernandez, G. (2009). Soigner le travail. Itinéraires d'un médecin du travail. Paris : Ères.

Fernandez, G. (2015). Gestes, action et analyse du travail. R. Ouvrier-Bonnaz, \& A. Weill-Fassina (Eds.), L'analyse du travail entre ruptures et évolutions (pp. 169-185). Toulouse : Octarès.

Fischer, S. L., Belbeck, A. L., \& Dickerson, C. R. (2010). The influence of providing feedback on force production and within participants reproducibility during maxima voluntary exertions for the anterior deltoid, middle deltoid and infraspinatus. Journal of Electromyography and Kinesiology, 20(1), 68-75. 
Gaudez, C., \& Aptel, M. (2008). Les mécanismes neurophysiologiques du mouvement, base pour la compréhension du geste. Le Travail Humain, 71(4), 385-404.

Gaudez, C., Bonnet, N., \& Aublet-Cuvelier, A. (2013, 28-30 août). Evaluations des astreintes : OREGE et Electromyographie de surface pour évaluer les facteurs de risque biomécaniques en situation réelle de travail. Paper presented at the In Actes du 48e Congrès de la Société d'Ergonomie de Langue Française, « Ergonomie \& Société, Quelles attentes ? Quelles réponses? », Paris.

Gaudez, C., \& Cail, F. (2016). Effects of mouse slant and desktop position on muscular and postural stresses, subject preference and performance in women aged 18-40 years. Ergonomics, 59(11), 1473-1486. https://doi.org/10.1080/00140139.2016.1148783

Gaudez, C., Wild, P., \& Aublet-Cuvelier, A. (2015). A better way of fitting clips? A comparative study with respect to physical workload. Applied Ergonomics, 51, 236-243. doi: https://doi.org/ 10.1016/j.apergo.2015.05.005

Jaquet, C. (2004). L'unité du corps et de l'esprit. Affects, actions et passions chez Spinoza. Paris : PUF.

Kelly, B. T., Kadrmas, W. R., Kirkendall, D. T., \& Speer, K. P. (1996). Optimal normalization tests for shoulder muscle activation: An electromyographic study. Journal of Orthopaedic Research, 14(4), 647-653.

Kloetzer, L., Quillerou-Grivot, E., \& Simonet, P. (2015). Engaging workers in WRMSD prevention: Two interdisciplinary case studies in an activity clinic. WORK: A Journal of Prevention, Assessment and Rehabilitation, 51(2), 161-173.

Koechlin, B. (1968). Techniques corporelles et leur notation symbolique. Langages, 10, 36-47. Retrieved from http://www.persee.fr/web/revues/home/prescript/article/ lgge_0458-726X_1968_num_3_10_2547

Leplat, J. (2005). Les automatismes dans l'activité : pour une réhabilitation et un bon usage. Activités, 2(2), 43-68. Retrieved from https://journals.openedition.org/activites/1797

Leplat, J. (2013). Les gestes dans l'activité en situation de travail. PISTES, 15(1). Retrieved from https://pistes.revues.org/2951 https://doi.org/10.4000/pistes.2951

Madeleine, P., \& Madsen, T. M. T. (2009). Changes in the amount and structure of motor variability during a deboning process are associated with work experience and neck-shoulder discomfort. Applied Ergonomics, 40(5), 887-894. https://doi.org/10.1016/j.apergo.2008.12.006

Madeleine, P., Voigt, M., \& Mathiassen, S. E. (2008). The size of cycle-to-cycle variability in biomechanical exposure among butchers performing a standardised cutting task. Ergonomics, 51(7), 1078-1095. https://doi.org/10.1080/00140130801958659

Massot, C., \& Simonet, P. (2017). Intervenir dans l'entreprise pour soutenir la discussion sur le travail comme moyen de transformation. PISTES, 19(3). Retrieved from http:// journals.openedition.org/pistes/5491; https://doi.org/10.4000/pistes.5491

Mathiassen, S. E., Möller, T., \& Forsman, M. (2003). Variability in mechanical exposure within and between individuals performing a highly constrained industrial work task. Ergonomics, 46(8), 800-824. https://doi.org/10.1080/0014013031000090125

Matkowski, B., Martin, A., \& Lepers, R. (2011). Comparison of maximal unilateral versus bilateral voluntary contraction force. European Journal of Applied Physiology, 111(8), 1571-1578. https:// doi.org/10.1007/s00421-010-1775-1 
McCully, S. P., Kumar, N., Lazarus, M. D., \& Karduna, A. R. (2005). Internal and external rotation of the shoulder: Effects of plane, end-range determination, and scapular motion. Journal of Shoulder and Elbow Surgery, 14(6), 602-610.

Mirzabekianz, E. (2000). Grammaire de la notation Benesh. Manuel élémentaire. Cahiers de pédagogie : Pantin : Ecole Nationale de Danse.

Oddone, I., Re, A., \& Briante, G. (1981). Redécouvrir l'expérience ouvrière. Vers une autre psychologie du travail ? Paris : Éditions sociales

Pezé, M. (1998). Les athlètes du quotidien : approche psychosomatique et psychodynamique des TMS. In ANACT (Ed.), TMS et évolution des conditions de travail. Les actes du séminaire Paris 98 (pp. 63-70). Retrieved from https://www.anact.fr/tms-et-evolution-des-conditions-de-travailseminaire-paris-1998.

Plamondon, A., \& Denis, D. (2008). Manutention : intérêt d'une approche conjointe ergonomiebiomécanique dans la compréhension du geste. Paper presented at the $2^{\mathrm{e}}$ Congrès francophone sur les troubles musculosquelettiques (TMS), Montréal, Canada.

Poussin, N., \& Simonet, P. (2017). La formalisation d'un répertoire des gestes de métier comme instrument de l'intervention du clinicien de l'activité : une question de recherche. In A.-

L. Ulmann, A. Weill-Fassina, \& T.H. Benchekroun (Eds.), Intervenir. Histoires, recherches, pratiques. Toulouse : Octarès Éditions.

Rivilis, I., Van Eerd, D., Cullen, K., Cole, D. C., Irvin, E., Tyson, J., \& Mahood, Q. (2008).

Effectiveness of participatory ergonomic interventions on health outcomes: A systematic review. Applied Ergonomics, 39(3), 342-358.

Roquelaure, Y. (2016). Promoting a Shared Representation of Workers' Activities to Improve Integrated Prevention of Work-Related Musculoskeletal Disorders. Safety and Health at Work, 7(2), 171-174. doi: https://doi.org/10.1016/j.shaw.2016.02.001

Savescu, A., \& Cuny-Guerrier, A. (2015). Multidisciplinary analysis of risk factors of MSD in meat cutting: the issue of organizing knife maintenance. Paper presented at the 19th Triennial Congress of the International Ergonomics Association, Melbourne, Australia.

Savescu, A., Gaudez, C., Aublet-Cuvelier, A., Simonet, P., Fernandez, G., Van-Trier, M., \& Clot, Y. (2010). Biomechanical metrology: a support in occupational controversies. Paper presented the PREMUS, Angers, France.

Savescu, A. \& Simonet, P. (2020). Pourquoi et comment associer la mesure biomécanique au cadre d'intervention de la clinique de l'activité dans une action de prévention des TMS ?, SELF2020, Paris, France

SENIAM5. (1997). The state of the art of sensors and sensor placement procedures for surface electromyography. A proposal for sensor placement procedures SENIAM project. In H. J. Hermens \& B. Freriks (Eds.), Copyright 1997 Roessing Research and Development

Silverstein, B., \& Clark, R. (2004). Interventions to reduce work-related musculoskeletal disorders. Journal of Electromyography and Kinesiology, 14(1), 135-152. https://doi.org/10.1016/ j.jelekin.2003.09.023

Simonet, P. (2009). L'examen méthodique d'un geste de métier pour une prévention durable des TMS : une intervention en clinique de l'activité. PISTES, 11(2). Retrieved from pistes website : www.pistes.uqam.ca

Simonet, P. (2011). L'hypo socialisation du mouvement. Prévention durable des troubles musculosquelettiques chez des fossoyeurs municipaux. PhD, Paris : CNAM. 
Simonet, P. (2012). La formation du geste entre standardisation et tensions du métier. In B. M. B. Prot (Ed.), Développer le pouvoir d'apprendre : pour une critique de la transmission en éducation et en formation (pp. 66-75). Bologne : TAO Digital Library.

Simonet, P., \& Caroly, S. (2008). Le développement des automatismes comme conception du geste professionnel pour une prévention durable des TMS. Paper presented at the $43^{\mathrm{e}}$ congrès de la Société d'Ergonomie de Langue Française, Ajaccio, France.

Simonet, P., \& Caroly, S., (2020). Geste dialogué et prévention des TMS. Le Travail Humain, 83(1), 1-32. https://doi.org/10.3917/th.831.0001.

Simonet, P., Caroly, S., \& Clot, Y. (2011). Méthodes d'observation de l'activité de travail et prévention durable des TMS : action et discussion interdisciplinaire entre clinique de l'activité et ergonomie. Activités, 8(1). https://doi.org/10.4000/activites.2481.

Simonet, P., \& Clot, Y. (2014). Qualité du travail, santé et clinique de l'activité. Méthode pour l'action. In E. Masson (Ed.), Encyclopédie médicochirurgicale, Pathologie professionnelle et de l'environnement. Paris.

Simonet, P., \& Poussin, N. (2014). La socialisation des gestes de métier : un enjeu de santé au travail qui interroge la formation. Revue éducation permanente, hors série AFPA Les synergies travailformation, 127-135.

Simonet, P., Savescu, A., Van Trier, M., Gaudez, C., \& Aublet-Cuvelier, A. (2011). La

pluridisciplinarité au service de la prévention des TMS : quand l'association entre psychologie du travail et biomécanique devient, pour les professionnels, support d'analyse des gestes de métier. from INRIA www.hal.inria.fr

St-Vincent, M., Chicoine, D., \& Beaugrand, S. (1998). Validation of a participatory ergonomic process in two plants in the electrical sector. International Journal of Industrial Ergonomics, 21(1), 11-21. doi : https://doi.org/10.1016/S0169-8141(97)00021-8

Thibault, J.-F., Merlin, X., \& Garrigou, A. (2013). De la production à l'usage de la mesure, quelle appropriation par deux entreprises industrielles ? Pour quelle prévention des TMS ? Pistes, 15(2). Retrieved from www.pistes.uqam.ca

Tomàs, J.-L. (2013). L'analyse psychologique du développement des gestes professionnels : une perspective pour la prévention des TMS ? Perspectives interdisciplinaires sur le travail et la santé, Pistes, 15(1).

Tomás, J.-L., Simonet, P., Clot, Y., \& Fernandez, G. (2009). Le corps : l'œuvre du collectif de travail. Corps, 6, 23-30.

Van Eerd, D., Cole, D., Irvin, E., Mahood, Q., Keown, K., Theberge, N., .Village, J., St Vincent, M., \& Cullen, K.L. (2010). Process and implementation of participatory ergonomic interventions: a systematic review. Ergonomics, 53(10), 1153-1166. https://doi.org/10.1080/00140139.2010.513452

Van Trier, M., Simonet, P., Fernandez, G., Savescu, A., \& Gaudez, C. (2010). Prévention durable des TMS chez des fossoyeurs de la ville de Paris. Paper presented at the $31^{\mathrm{e}}$ Congrès National de Médecine et Santé au Travail, Toulouse, France.

Vézina, N. (2001). La pratique de l'ergonomie face aux TMS : ouverture à l'interdisciplinarité. Paper presented at the Société d'Ergonomie de Langue Française-Association Canadienne d'Ergonomie (SELF-ACE), Monréal, Canada.

Vézina, N., Prevost, J., Lajoie, A., \& Beauchamp, Y. (1999). Elaboration d'une formation à l'affilage des couteaux : Le travail d'un collectif, travailleurs et ergonomes. Pistes, 1(1). Retrieved from http://www.pistes.uqam.ca. 
Vinck, D. (2002). Fonctions et modèles pour l'interdisciplinarité en ergonomie. Performances humaines et techniques, 5, 7-13.

Vygotski, L. (1927/1999). La signification historique de la crise en psychologie. Neufchâtel.

Vygotski, L. (1934/1994). Défectologie et déficience mentale. Neuchâtel : Delachaux et Niestlé.

Vygotski, L. (1978). Mind in society. The development of higher psychological process. Cambridge \& London : Harvard University Press.

Vygotsky, L. S. (1997). The instrumental method in psychology. The collected works of L. S. Vygotsky. vol. 3. Problems of the theory and history of psychology. New York : Plenum.

Wallon, H. (1942/1970). De l'acte à la pensée. Paris : Flammarion.

Wisner, A. (1997). Aspects psychologiques de l'anthropotechnologie. Le Travail Humain, 60(3), 229-254.

Yang, J. F., \& Winter, D. A. (1983). Electromyography reliability in maximal and submaximal isometric contractions. Archives of Physical Medicine and Rehabilitation 64, 417-420.

Zipp, P. (1982). Recommendations for the standardization of lead positions in surface electromyography. European Journal of Applied Physiology, 50, 41-45.

\section{NOTES}

1. Au travail, le geste personnel est toujours adressé aux collègues destinataires des efforts consentis. Le métier est à ce titre interpersonnel. Il vit donc - ou il meurt - entre professionnels et en chacun d'eux dans la motricité des échanges personnels et interpersonnels sur ce qui est à faire ou à refaire, à dire ou à redire. Il n'est pas tout entier dans le présent des activités partagées en situation : c'est l'histoire et la mémoire collective qui donnent à chacun le répondant pour agir dans le présent et « voir venir » le futur. Cette mémoire du métier est transpersonnelle, personne n'en est propriétaire. C'est le clavier collectif des gestes et des mots sur lequel chaque sujet peut jouer sa petite musique, le genre professionnel à styliser par chacun. Mais le métier existe aussi, impersonnel, consigné dans des fonctions officielles prescrites: une carrière, une retraite, des tâches, un statut, une formation, des critères d'évaluation et de recrutement, des indicateurs standards de performance. Ce métier impersonnel est psychologiquement vital pour imaginer ce qu'on peut devenir ou pas, ce qu'on pourrait faire au-delà de ce qu'on fait déjà. Des tensions se faufilent partout dans cette architecture, jusqu'à parfois la faire exploser (Simonet \& Clot, 2014).

2. Nous mobilisons une intervention-recherche sur laquelle nous avons déjà eu l'occasion de prendre appui pour discuter différentes thématiques d'intervention et de recherche en lien avec la prévention des TMS (Simonet, 2009 ; Simonet, Savescu, Van Trier, Gaudez, \& Aublet-Cuvelier, 2011) et plus largement de santé et de transformation de l'organisation du travail (Massot \& Simonet, 2017 ; Poussin \& Simonet, 2017 ; Simonet \& Clot, 2014). Dans cet article nous insisterons spécifiquement sur l'association interdisciplinaire entre l'analyse biomécanique et le cadre méthodologique clinique de l'activité dans l'histoire de cette intervention (Savescu et al., 2010 ; Simonet et al., 2011) mais aussi dans celle d'un dialogue scientifique engagé (Clot \& Fernandez, 2005).

3. Le comité de pilotage sous la direction du chef de service des cimetières réunit les personnels de l'encadrement, de la médecine du travail, de la prévention des risques professionnels et les intervenants-chercheurs.

4. Cette intervention a permis de reposer la question de l'usage d'excavatrice mécanique dans les cimetières entre les décideurs réunis en comité de pilotage et, à leur demande, un nouveau 
dispositif de formation interne co-construit avec les fossoyeurs a été initié sous l'impulsion du CHSCT.

5. Cette période permet aux participants volontaires de poser toutes leurs questions concernant les moyens de mesure. De même, ils sont encouragés d'arrêter leur participation s'ils ressentent une gêne particulière.

6. En suivant Cosnier pour qui « le discours vivant est le produit d'un corps parlant » (Cosnier, 1996) il nous est apparu nécessaire de rendre visibles les articulations entre expériences sensorimotrices et échanges d'arguments dans le cours du dialogue sans proposer ni une classification des gestes dont les nombreuses limites sont connues depuis longtemps (Koechlin, 1968) ni même une quelconque théorie sémiotique du geste sans rapport avec l'activité argumentative.

7. Cette opération de décontextualisation du geste de l'action ordinaire est une voie de recherche et d'action qui interroge les liens entre geste, action et analyse du travail (Fernandez, 2015). Rappelons que ce geste de jeté arrière réalisé au cimetière est re-présenté mais sous une forme dénaturée par les images de la vidéo qui présente une série de jetés arrières accolés après avoir été définis par un début et une fin éliminant tout une partie des évènements de l'activité réelle de travail. Mais cette transformation de la réalité était voulue dans ce dispositif. Elle cherchait à concentrer l'attention des fossoyeurs sur l'exécution de ce geste et à provoquer chez eux un niveau plus fin d'observations, d'étonnements et d'interrogations. Le jeté arrière examiné aussi finement est devenu peu à peu l'objet du dialogue entre les deux professionnels et un moyen d'argumentation pour discuter les mérites comparés de la manière dont chacun l'exécute et audelà de l'engagement de leur corps dans l'activité du creusement tout en expérimentant de nouvelles sensations kinesthésiques.

\section{RÉSUMÉS}

En France et à l'international, les troubles musculo-squelettiques (TMS) restent les maladies professionnelles les plus répandues. La littérature scientifique a établi l'existence de liens entre la survenue des TMS et la réalisation des gestes en situation de travail. L'objectif de cet article est de montrer comment l'analyse biomécanique participe à la création d'outils d'intervention mis en œuvre dans un cadre méthodologique en clinique de l'activité dans une optique de prévention des TMS se basant sur l'analyse du geste. La méthodologie interdisciplinaire mise en œuvre auprès de huit fossoyeurs comprend plusieurs étapes incluant une analyse de l'activité musculaire des muscles de l'épaule et du dos laquelle est utilisée comme support à des autoconfrontations. Les résultats montrent que la méthodologie interdisciplinaire permet d'ouvrir plus largement la zone de développement potentiel du geste dans l'activité des fossoyeurs en stimulant leurs discussions professionnelles. La mise en visibilité et en discussion dans le métier de la variabilité inter et intra-individuelle de réalisation d'un même geste au contact des questions concrètes de travail a ouvert le milieu professionnel vers de nouvelles formes d'actions de prévention des TMS dans l'organisation prescrite du travail.

In both France and abroad, musculoskeletal disorders (MSD) remain the most widespread occupational diseases. The scientific literature has established that links exist between the occurrence of MSD and the professional gesture. The aim of this paper is to show how biomechanical analysis helps to create intervention tools implemented in a methodological framework in clinical activity with a view to preventing MSD through analysis of the gesture. The 
aim of this paper is to analyse how an intervention was conducted within the general methodological framework of occupational psychology using activity-clinic research based on professional gesture analysis and it also benefited from interdisciplinary co-operation with biomechanics. The interdisciplinary methodology employed in relation to eight gravediggers consisted of different steps that included a detailed analysis of the muscular activity of the shoulder and back muscles. The results show that the interdisciplinary methodology makes it possible to widen the potential development zone of the gesture in the gravediggers' activity by stimulating their professional discussions. The creation of visibility and discussion in the profession of inter- and intra-individual variability has opened up the professional world to new forms of action to prevent MSD.

\section{INDEX}

Mots-clés : geste, biomécanique, clinique de l'activité, méthodologie, prévention, troubles musculosquelettiques

Keywords : gesture, biomechanics, occupational psychology, methodology, prevention, musculoskeletal disorders

\section{AUTEURS}

\section{ADRIANA SAVESCU}

Laboratoire Physiologie-Mouvement-Travail, Institut National de Recherche et de Sécurité, CS 60027, 54519 Vandœuvre-lès-Nancy Cedex, France

adriana.savescu@inrs.fr

\section{PASCAL SIMONET}

Centre de Recherche en Éducation de Nantes (CREN, EA 2661), Université de Nantes, BP 81227, 44312 Nantes Cedex 3

pascal.simonet@univ-nantes.fr

\section{CLARISSE GAUDEZ}

Laboratoire Physiologie-Mouvement-Travail, Institut National de Recherche et de Sécurité, CS 60027, 54519 Vandœuvre-lès-Nancy Cedex, France

\section{GABRIEL FERNANDEZ}

Équipe Psychologie du travail et clinique de l'activité, Centre de recherche sur le travail et le développement (CRTD, EA 4132), Conservatoire national des arts et métiers, 41, rue Gay Lussac, 75 005, Paris 